\title{
A Comparative Study of Goodness-of-Fit Tests for the Laplace Distribution
}

\author{
Apostolos Batsidis \\ University of Ioannina
}

\author{
Polychronis Economou \\ University of Patras
}

\author{
Shaul K. Bar-Lev \\ HIT - Holon Institute \\ of Technology
}

\begin{abstract}
The Laplace distribution is one of the earliest distributions in probability theory and is a frequently used distribution in many fields. Consequently, various goodness-of-fit tests for the Laplace distribution have been thoroughly derived in the literature. The purpose of this paper is to carry out a comparative study of these tests as well as a new one we develop. Power comparisons of all such tests are performed via Monte Carlo simulations of sample data generated from twenty seven alternatives distributions. Despite the fact that no single test was found to be most powerful in all situations, several useful recommendations however are made.
\end{abstract}

Keywords: Laplace distribution, Monte Carlo study, goodness-of-fit.

\section{Introduction}

The Laplace or the double exponential distribution introduced by Laplace (1774) is one of the earliest distribution discussed in probability theory. It is a symmetric distribution which has been used as an alternative to the normal distribution in robustness studies and in modeling phenomena with heavier than normal tails (see Kozubowski and Nadarajah (2010), Cordeiro and Lemonte (2011) and references therein). The areas in which the Laplace distribution has been used are rather wide. A detailed list of these areas along with some references can be found in Kotz, Kozubowski, and Podgorski (2001), Johnson, Kotz, and Balakrishnan (1995), Kozubowski and Nadarajah (2010) and Cordeiro and Lemonte (2011).

Due to its importance in modeling real data, validating the assumption of Laplace distribution has been of great concern. Indeed, numerous studies have been devoted to testing methods for detecting a departure from Laplace. The existing tests can be classified into five classes, namely (i) tests based on the empirical distribution function (c.f. Yen and Moore 1988; Rublík 1997; Puig and Stephens 2000a; Chen 2002, and references cited therein); (ii) tests based on the empirical characteristic function (c.f. Meintanis 2005); (iii) moment based tests, i.e., tests based on sample moments, skewness and kurtosis (González-Estrada and Villaseñor 2016; Gel 2010; Rayner and Best 1989; Langholz and Kronmal 1991; Li and Papadopoulos 2002); (iv) entropy and divergence based tests (Choi and Kim 2006; Rizzo and Haman 2016; Alizadeh Noughabi and Balakrishnan 2016; Alizadeh Noughabi 2019; Alizadeh Noughabi and 
Park 2016) (v) other tests (Gulati 2011).

The main goal of this paper is to present a detailed comparison of the various testing procedures for detecting departures from the Laplace distribution. In this context, the rest of the paper is organized as follows. In Section 2 we present some preliminaries on the Laplace distribution. In Section 3 the existing goodness-of fit tests for the Laplace distribution are briefly presented and classified into one of the five classes mentioned above. Moreover, a new modification of the moment structure based test presented in Li and Papadopoulos (2002, p.74) for testing departure from Laplace is presented and studied in detail. Section 4 is devoted to executing Monte Carlo simulations for comparing the goodness-of fit tests for the Laplace distribution described in Section 3. Such a comparison includes 22 tests available in the literature with 27 possible distributions (symmetric or asymmetric) presented as alternatives to the Laplace distribution. To our best knowledge this study is the first attempt made in the literature to compare so many goodness-of-fit tests for the Laplace distribution and alternative distributions (c.f., Best, Rayner, and Thas (2008) and Gel (2010)). Section 5 presents a summary of the results of the paper and some practical relevant conclusions. All proofs related to the new goodness-of fit test for the Laplace distribution introduced in Section 3 appear in an Appendix. $\mathrm{R}$ codes used to showing and demonstrating the computational and numerical parts of this paper along with their implementation are also presented in the Appendix.

\section{Background}

In this section we present some preliminaries and notation related to the Laplace distribution. The classical Laplace distribution $\mathcal{C} L(\delta, c)$ has a two-parameter probability density function (p.d.f) and cumulative distribution function (c.d.f) given by

$$
f_{0}(x ; \delta, c)=\frac{1}{2 c} e^{-\frac{|x-\delta|}{c}}, \text { with } \delta \in R, c>0, x \in R,
$$

and

$$
F_{0}(x ; \delta, c)=0.5+0.5 \operatorname{sgn}(x-\delta)\left(1-e^{-\frac{|x-\delta|}{c}}\right),
$$

where $\operatorname{sgn}(x-\delta)$ equals $-1,0$, or 1 , depending on whether $x-\delta$ is negative, zero, or positive, respectively. The case $\mathcal{C} L(0,1)$ with $\delta=0$ and $c=1$ is called the classical standard Laplace distribution.

In the sequel we denote, respectively, by $\mu_{l}=E\left(X^{l}\right)=\int x^{l} d F(x)$ and $k_{l}=E\left[\left(X-\mu_{1}\right)^{l}\right]=$ $\int\left(x-\mu_{1}\right)^{l} d F(x), l \in \mathbb{N}$, the $l$-th moment and $l$-th central moment of a r.v. $X$ with c.d.f. $F$, while $\sqrt{\beta_{1}}=k_{3} / k_{2}^{3 / 2}$ and $\beta_{2}=k_{4} / k_{2}^{2}$ are used to denote the corresponding skewness and kurtosis. For the $\mathcal{C} L(\delta, c)$, the mean, median and mode are all equal to $\delta$, the variance to $2 c^{2}$, while the skewness and kurtosis are 0 and 6 , respectively.

Let $\left(X_{1}, \ldots, X_{n}\right)$ be a random sample of size $n$ and $X_{(1)} \leq X_{(2)} \leq \ldots \leq X_{(n)}$ denote its order statistic arrangement. If this sample is taken from a $\mathcal{C} L(\delta, c)$ population then the maximum likelihood estimators (mle) of $\delta$ and $c$ are

$$
\hat{\delta}_{n}:=\hat{\delta}_{n}\left(X_{1}, \ldots, X_{n}\right)=\operatorname{Median}\left(X_{1}, \ldots, X_{n}\right)=\left\{\begin{array}{ll}
X_{(n+1) / 2}, & \text { if } n \text { is odd } \\
\frac{X_{(n / 2)}+X_{(n / 2+1)}}{2}, & \text { if } n \text { is even }
\end{array},\right.
$$

and

$$
\hat{c}_{n}:=\hat{c}_{n}\left(X_{1}, \ldots, X_{n}\right)=\frac{1}{n} \sum_{i=1}^{n}\left|X_{i}-\hat{\delta}_{n}\right| .
$$

By using the fact that $E(X)=\delta$ and $\operatorname{Var}(X)=2 c^{2}$ then the corresponding method of moments estimators (mom) of $\delta$ and $c$ are

$$
\tilde{\delta}_{n}:=\tilde{\delta}_{n}\left(X_{1}, \ldots, X_{n}\right)=\bar{X}_{n}
$$


and

$$
\tilde{c}_{n}:=\tilde{c}_{n}\left(X_{1}, \ldots, X_{n}\right)=\sqrt{\frac{S_{n}^{2}}{2}},
$$

where

$$
S_{n}^{2}=\frac{1}{n} \sum_{i=1}^{n}\left(X_{i}-\bar{X}_{n}\right)^{2} .
$$

The mle $\hat{\delta}_{n}$ and the mom estimator $\tilde{\delta}_{n}$ of $\delta$ are affine equivariant, i.e., for any real $d$ and $\beta>0$

$$
\hat{\delta}_{n}\left(\beta X_{1}+d, \ldots, \beta X_{n}+d\right)=\beta \hat{\delta}_{n}\left(X_{1}, \ldots, X_{n}\right)+d,
$$

and

$$
\tilde{\delta}_{n}\left(\beta X_{1}+d, \ldots, \beta X_{n}+d\right)=\beta \tilde{\delta}_{n}\left(X_{1}, \ldots, X_{n}\right)+d .
$$

Moreover, the mle $\hat{c}_{n}$ and the mom estimator $\tilde{c}_{n}$ of $c$ are, respectively, location invariant and scale equivariant, i.e., for any real $d$ and $\beta>0$

$$
\hat{c}_{n}\left(\beta X_{1}+d, \ldots, \beta X_{n}+d\right)=\beta \hat{c}_{n}\left(X_{1}, \ldots, X_{n}\right),
$$

and

$$
\hat{c}_{n}\left(\beta X_{1}+d, \ldots, \beta X_{n}+d\right)=\beta \hat{c}_{n}\left(X_{1}, \ldots, X_{n}\right) .
$$

In the rest of the paper we shall use the following notation:

$$
\begin{aligned}
& Z_{i}=F_{0}\left(X_{i} ; \hat{\delta}_{n}, \hat{c}_{n}\right), Z_{(i)}=F_{0}\left(X_{(i)} ; \hat{\delta}_{n}, \hat{c}_{n}\right), Y_{i}=\frac{X_{i}-\delta}{c} \\
& \hat{Y}_{i}=\frac{X_{i}-\hat{\delta}_{n}}{\hat{c}_{n}}, \tilde{Y}_{i}=\frac{X_{i}-\tilde{\delta}_{n}}{\tilde{c}_{n}}, \hat{U}_{(i)}=\left|\hat{Y}_{(i)}\right|, \text { and } \hat{V}_{i}=X_{i}-\hat{\delta}_{n} \text { for } i=1, \ldots, n .
\end{aligned}
$$

\section{Goodness-of-fit tests for the Laplace distribution}

Let $\left(X_{1}, \ldots, X_{n}\right)$ be a random sample of size $n$ taken from a c.d.f. $F(x)$ and p.d.f $f(x)$. At a significance level $\alpha$ we consider testing the null hypothesis that the parent distribution is Laplace vs any other alternative, i.e.,

$$
H_{0}: F(\cdot)=F_{0}(\cdot ; \delta, c) \text {, for some } \delta \in R \text { and } c>0,
$$

vs.

$$
H_{1}: F \text { is } \operatorname{not} \mathcal{C} L(\delta, c) .
$$

As if $X \sim \mathcal{C} L(\delta, c)$ then $\beta X+d \sim \mathcal{C} L(\beta \delta+d, \beta c)$ for any $d \in \mathbb{R}$ and $\beta \in R^{+}$it follows that the family of Laplace distributions is invariant under affine transformations $X \rightarrow \beta X+d$. Thus any test statistic, say $H_{n}\left(X_{1}, \ldots, X_{n}\right)$, used for testing departure from the Laplace distribution should also be affine invariant (see Meintanis (2005), p.927). Accordingly, if $\left(X_{1}, \ldots, X_{n}\right)$ is a random sample from $\mathcal{C} L(\delta, c)$ then the following relation should hold

$$
H_{n}\left(\beta X_{1}+d, \ldots, \beta X_{n}+d\right)=H_{n}\left(X_{1}, \ldots, X_{n}\right) .
$$

In the sequel, the existing goodness-of fit tests for the Laplace distribution are briefly presented. Moreover, a new modification of the moment structure based test presented in Li and Papadopoulos (2002, p.74) for testing departure from the Laplace distribution is presented and studied in detail. The modification is required as the test proposed by Li and Papadopoulos (2002) is not affine invariant.

As we previously indicated all existing tests are classified into five classes. 


\subsection{Tests based on the empirical distribution function}

The key idea of the empirical distribution function (e.d.f.) tests is to compare the data estimated c.d.f. with the hypothesized c.d.f. Thus e.d.f. tests are based on discrepancy measures between the c.d.f. of the Laplace distribution given in (2) with $\delta$ and $c$ being estimated appropriately and the e.d.f. defined by

$$
F_{n}(x)=\frac{\sum_{i=1}^{n} I\left(X_{i} \leq x\right)}{n}=\frac{\# \text { of observations } \leq x}{n},-\infty<x<\infty,
$$

where $I(\cdot)$ is the indicator function. In this frame, five different e.d.f. tests have been presented and studied in the literature, namely the Cramer-von Mises $\left(W^{2}\right)$, the Watson $\left(U^{2}\right)$, the Anderson-Darling $\left(A^{2}\right)$, the Kolmogorov-Smirnov $(\sqrt{n} D)$ and the Kuiper $(V)$ tests (c.f. Yen and Moore 1988; Puig and Stephens 2000a; Chen 2002, and references cited therein). The test statistics $W^{2}, U^{2}$ and $A^{2}$ belong to the Cramer von Mises family, while the other two to the Kolmogorov-Smirnov family of tests. These test statistics have the form

$$
\begin{gathered}
W^{2}=\frac{1}{12 n}+\sum_{i=1}^{n}\left(Z_{(i)}-(2 i-1) /(2 n)\right)^{2}, \\
U^{2}=W^{2}-n\left(\bar{Z}_{n}-0.5\right)^{2}, \\
A^{2}=-n-\frac{1}{n} \sum_{i=1}^{n}\left((2 i-1) \log \left(Z_{(i)}\right)+(2(n-i)+1) \log \left(1-Z_{(i)}\right)\right), \\
D=\max \left\{D^{+}, D^{-}\right\} \text {and } V=D^{+}+D^{-},
\end{gathered}
$$

where

$$
D^{+}=\max _{i=1, . ., n}\left(\frac{i}{n}-Z_{(i)}\right) \text { and } D^{-}=\max _{i=1, . ., n}\left(Z_{(i)}-\frac{i-1}{n}\right)
$$

with the $Z_{(i)}$ being defined in Section 2 .

All of the above tests are right tailed, i.e., the null hypothesis is rejected for large values. Therefore the critical values are the $100(1-\alpha)$-th percentiles of the empirical distribution of the respective test statistic. Asymptotic critical values are also available for the tests belonging to the Cramer von Mises family (see Puig and Stephens (2000a)). A more detailed discussion, asymptotic results on the e.d.f. tests and tables of critical values based on 50,000 Monte Carlo samples of size $n$ for specific values of $n$ can be found in Puig and Stephens (2000a).

\subsection{Tests based on the empirical characteristic function}

The characteristic function (c.f.) of $X \sim \mathcal{C} L(\delta, c)$ is

$$
\phi_{0}(t ; \delta, c)=\frac{\exp (i \delta t)}{1+c^{2} t^{2}}, t \in \mathbb{R} .
$$

Meintanis (2005) proposed a class of goodness-of-fit tests for the Laplace distribution based on its c.f. More specifically, the key idea behind his proposal is related to the fact that the c.f. of the Laplace distribution satisfies the relation

$$
\left(1+c^{2} t^{2}\right) \phi(t)-\exp (i \delta t)=0, t \in \mathbb{R} .
$$

Under the null hypothesis of Laplace distribution then for large $n$ the transformed data $\hat{Y}_{i}$ or $\tilde{Y}_{i}$ are approximately $\mathcal{C} L(0,1)$. By using these transformed data, Meintanis (2005) proposed a 
weighted integral of the squared of an empirical counterpart of the equation (15) for $c=1$ and $\delta=0$. To be more specific, Meintanis (2005) proposed the following classes of test statistics

$$
T_{n}^{j}=n \int_{-\infty}^{\infty}\left|\left(1+t^{2}\right) \phi_{n}^{j}(t)-1\right|^{2} w(t) d t, j=M L, M O
$$

where $w(t)$ denotes an appropriate weight function, $\phi_{n}^{M L}(t)$ and $\phi_{n}^{M O}(t)$ are the empirical characteristic functions (e.c.f.) of the transformed data $\hat{Y}_{i}$ and $\tilde{Y}_{i}$, respectively, defined by

$$
\phi_{n}^{M L}(t)=\frac{\sum_{i=1}^{n} \exp \left(i t \hat{Y}_{i}\right)}{n} \text { and } \phi_{n}^{M O}(t)=\frac{\sum_{i=1}^{n} \exp \left(i t \tilde{Y}_{i}\right)}{n}
$$

For computational purposes Meintanis (2005) focused on two parametric classes of weight functions for which both $T_{n}^{M L}$ and $T_{n}^{M O}$ take simple forms. In this frame, the test statistics corresponding to $w(t)=\exp (-a|t|), a>0$ are denoted by $T_{n, a}^{(1, M O)}$ or $T_{n, a}^{(1, M L)}$. Similarly, the test statistics corresponding to $w(t)=\exp \left(-a t^{2}\right), a>0$, are denoted by $T_{n, a}^{(2, M O)}$ or $T_{n, a}^{(2, M L)}$. For simple forms of these statistics and further computational details we refer to $\mathrm{p}$. 928 in Meintanis (2005). Based on the simulation study performed by Meintanis (2005) it is recommended to use $T_{n, 2}^{(1, M O)}, T_{n, 2}^{(1, M L)}, T_{n, 0.5}^{(1, M O)}$ and $T_{n, 0.5}^{(2, M L)}$. Indeed, for this reason we use these statistics in our simulation study.

All the above tests are again right tailed. The appropriate critical values which are the $100(1-\alpha)$-th percentiles of the empirical distribution of the respective test statistics are given in Meintanis (2005). A further detailed discussion, asymptotic results on the e.c.f. tests and tables of critical values based on 100,000 Monte Carlo samples of size $n=20,50$ can be found in Meintanis (2005).

\subsection{Sample moments based procedures}

Moment-based procedures are widely common for testing departures from a hypothesized distribution. In this section some existing tests are briefly presented, while a new modification of the moment structure based test presented in Li and Papadopoulos (2002, p.74) for testing a departure from the Laplace distribution is presented and studied in detail.

Best et al. tests

Smooth tests of goodness of fit, described in Rayner and Best (1989), seek to assess the fit of the data to a given p.d.f. $f(x ; \theta)$ within the following class of alternatives of order $k$ given by

$$
g_{k}(x ; \theta, \beta)=C(\theta, \beta) \exp \left(\sum_{i=1}^{k} \beta_{i} h_{i}(x ; \theta)\right) f(x ; \theta) .
$$

Here, $\theta$ is a vector of unknown parameters, $C(\theta, \beta)$ is a normalizing constant and $h_{i}(x ; \theta), i=$ $1, \ldots, k$, is a set of functions which are orthonormal on the hypothesized distribution $f(x ; \theta)$. If $E_{0}$ denotes the expectation when the model that generates the data is $f(x ; \theta)$ then orthonormality means that $E_{0}\left[h_{r}(X ; \theta) h_{s}(X ; \theta)\right]=\delta_{r s}$ for $r, s=0,1, \ldots$, where $\delta_{r s}=1$ if $r=s$ and $\delta_{r s}=0$ if $r \neq s$. It is obvious that the alternatives are characterized by their order, i.e., the greater the order $k$ the richer is the class of alternatives.

In this frame, based on a sample $X_{1}, \ldots, X_{n}$, if $\widetilde{\theta}_{n}\left(X_{1}, \ldots, X_{n}\right)$ is either the mle or the moment based estimator of the unknown parameters $\theta$ of the hypothesized distribution, Rayner and Best (1989) proposed a goodness-of-fit test of a hypothesized distribution using

$$
V_{r}=\sum_{i=1}^{n} h_{r}\left(X_{i} ; \widetilde{\theta}_{n}\left(X_{1}, \ldots, X_{n}\right)\right) / \sqrt{n}
$$


Motivated by Rayner and Best (1989) and under the moment estimation method when utilizing the complete orthonormal functions for $r=3$, 4, Best et al. (2008) proposed the following two smooth goodness-of fit tests for the Laplace distribution:

$$
V_{3}=\sqrt{n b_{1} / 54} \text { and } V_{4}=\left(b_{2}-6\right) \sqrt{n / 1072.8}
$$

where

$$
\sqrt{b_{1}}=\frac{\sum_{i=1}^{n}\left(X_{i}-\bar{X}_{n}\right)^{3}}{n S_{n}^{3}} \text { and } b_{2}=\frac{\sum_{i=1}^{n}\left(X_{i}-\bar{X}_{n}\right)^{4}}{n S_{n}^{4}}
$$

and $S_{n}$ is defined in (7). Here, $\sqrt{b_{1}}$ and $b_{2}$ are estimators of the population skewness and kurtosis, respectively, based on method of moments.

Asymptotically, both statistics follow a normal distribution with mean zero and variance $7 / 6$ and 165/149, respectively (see Best et al. (2008)). However Best et al. (2008) concluded that in practice the use of the asymptotic critical values is not recommended as the the convergence is slow. Taking into account that the null hypothesis of Laplace for both tests is rejected for large absolute values (two-tailed tests), then the critical values are the $100(\alpha / 2)$-th and $100(1-\alpha / 2)$-th percentiles of the empirical distribution of the test statistics.

\section{Gel tests}

Gel (2010) proposed goodness-of-fit procedures for the Laplace distribution based on alternative estimates of population skewness and kurtosis obtained by utilizing the mle of the unknown variance in the denominator of the population skewness and kurtosis. Specifically, the $K$ test by Gel (2010) is defined as

$$
K=\frac{n}{C_{1}}\left(\sqrt{u_{1}}\right)^{2}+\frac{n}{C_{2}}\left(u_{2}-6\right)^{2}, C_{1}, C_{2}>0,
$$

where

$$
\sqrt{u_{1}}=\frac{n^{-1} \sum_{i=1}^{n}\left(X_{i}-\bar{X}_{n}\right)^{3}}{\left(\sqrt{2} \hat{c}_{n}\right)^{3}} \text { and } u_{2}=\frac{n^{-1} \sum_{i=1}^{n}\left(X_{i}-\bar{X}_{n}\right)^{4}}{\left(\sqrt{2} \hat{c}_{n}\right)^{4}},
$$

with $\hat{c}_{n}$ being the mle of $c$ given in (4) while $C_{1}$ and $C_{2}$ are the asymptotic variances of $\sqrt{n u_{1}}$ and $\sqrt{n} u_{2}$, respectively. Gel (2010) proved that under the Laplace distribution, then $K$ follows asymptotically a chi-square distribution with two degrees of freedom. Here the null hypothesis of Laplace distribution is rejected if $K \geq \chi_{1-\alpha, 2}^{2}$ where $\chi_{1-\alpha, 2}^{2}$ is the upper a percentile of the chi-square distribution with two degrees of freedom. The constants $C_{1}$ and $C_{2}$ can be obtained using the multivariate Taylor-expansions. However, as these calculations are rather cumbersome, Gel (2010) recommended for small or moderate samples to use $C_{1}=60$ and $C_{2}=1200$ or to approximate them based on functions given in p. 960 by Gel (2010). Also, based on a Monte Carlo study by Gel (2010), the use of asymptotic critical values for small to moderately large samples is not recommended. Instead the use of the empirical critical values is recommended in which case the choice of $C_{1}$ and $C_{2}$ does not play any role. In this frame, taking into account that the null hypothesis of Laplace distribution is rejected for large values of $K$ - implying a right tailed test - the critical values are taken to be 100(1- $\alpha)$-th percentiles of the empirical distribution of the respective test statistic. For more details on the $K$ tests as well as to individual tests based on $\sqrt{u_{1}}$ and $u_{2}$ see Gel (2010).

\section{A ratio gof tests}

González-Estrada and Villaseñor (2016) proposed two tests based on ratio of estimators for the scale parameter of the Laplace distribution. To be more specific, the first test statistic denoted by $R_{n}$ is defined as the ratio of the sample mean absolute deviation around the sample mean to the moment estimator of $c$, while the second test denoted by $R_{n}^{\prime}$ is defined as the ratio of the sample mean absolute deviation around the sample mean to the mle of $c$, i.e.,

$$
R_{n}=\frac{\sqrt{2} \sum_{i=1}^{n}\left|X_{i}-\bar{X}_{n}\right|}{n S_{n}} \text { and } R_{n}^{\prime}=\frac{\sum_{i=1}^{n}\left|X_{i}-\bar{X}_{n}\right|}{\sum_{i=1}^{n}\left|X_{i}-\hat{\delta}_{n}\right|}
$$


where $S_{n}^{2}$ is defined in (7) and $\hat{\delta}_{n}$ is the mle of $\delta$ given in (3).

The idea behind the two tests is that under the null hypothesis of Laplace distribution the test statistics are expected to take values close to one. Thus both test are two-tailed tests. González-Estrada and Villaseñor (2016) proved that under the hypothesis of Laplace distribution $\sqrt{4 n}\left(R_{n}-1\right)$ follows asymptotically a standard normal distribution. Thus the null hypothesis is rejected if $\left|\sqrt{4 n}\left(R_{n}-1\right)\right| \geq z_{\alpha / 2}$, where $z_{\alpha / 2}=\Phi^{-1}(1-\alpha / 2), \Phi^{-1}(\cdot)$ is the quantile function of the $N(0,1)$ distribution. In the simulation study of the next section we use the $100(\alpha / 2)$-th and the 100(1-a/2)-th percentiles of the empirical distribution of the test statistics. For more details on $R_{n}$ and $R_{n}^{\prime}$ tests see González-Estrada and Villaseñor (2016).

\section{Langholz and Kronmal test}

Goodness-of-fit tests for the Laplace distribution can obtained as a special case of the class of tests proposed by Langholz and Kronmal (1991). The key idea behind their method is motivated by the fact that when the hypothesized distribution $F$ is completely specified then $X \sim F$ if and only if $F(X) \sim U(0,1)$. Their approach compares then the estimated Fourier coefficients to those of the $U(0,1)$ density. For the special case of Laplace, their test statistic based on the first Fourier coefficient in the density estimation procedure of Fellner (1974) can be written in the following form

$$
K_{1}=2.26 n\left(\hat{C}^{2}+\hat{S}^{2}\right)
$$

where

$$
\hat{C}=n^{-1} \sum_{i=1}^{n} \cos \left(2 \pi Z_{i}^{\prime}\right) \text { and } \hat{S}=n^{-1} \sum_{i=1}^{n} \sin \left(2 \pi Z_{i}^{\prime}\right)
$$

are the estimated first trigonometric moments, with $Z_{i}^{\prime}=F_{0}\left(X_{i} ; \tilde{\delta}_{n}, \tilde{c}_{n}\right)$. Under the hypothesis of Laplace, $K_{1}$ follows asymptotically a chi-square distribution with two degrees of freedom. Since the appropriate test is right-tailed we shall use in our simulation section the empirical critical values being the 100 $(1-\alpha)$-th percentiles of the empirical distribution. For more details on the $K_{1}$ test see Langholz and Kronmal (1991).

\section{A modification of a test based on moment structure}

A general and interesting method for testing a departure from a given parametric family of distributions was proposed by $\mathrm{Li}$ and Papadopoulos (2002). Their idea is mainly simple as it is based on some moment structure relation holding among the members of the respective parametric family of distributions under the null hypothesis. Then based on such a relation, a test statistic is proposed for testing departures from the relevant family. Li and Papadopoulos (2002) demonstrated their approach for some common parametric families of distributions. Among them is the Laplace family for which a test was proposed but was not studied in details. In the sequel, we present a modification of such a test by requiring that it will be affine invariant and study it in details.

If $F$ is $\mathcal{C} L(\delta, c)$ then it can be simply seen that

$$
g\left(\mu_{1}, \mu_{2}, \mu_{3}\right) \doteq \mu_{3}-3 \mu_{1} \mu_{2}+2 \mu_{1}^{3}=0, \text { for any } \delta \text { and } c .
$$

This would imply that if the relation in (22) does not hold then the sample is not taken from $\mathcal{C} L(\delta, c)$. The reverse statement is obviously incorrect as there might exist some other distributions for which (22) holds. Such a test is termed in the literature (c.f. Fang, Zhu, and Bentler 1993; Liang, Fang, and Hickernell 2008; Batsidis and Zografos 2013) as a 'necessary' (but not sufficient). Necessary tests imply that small p-values (say, less than 5\%) of the tests indicate evidence for departure from the family of distributions under the null hypothesis 
whereas larger p-values imply that no sufficient evidence is available for drawing any other statistical conclusion.

Indeed, a necessary test based on (22) for testing $H_{0}$ vs. $H_{1}$ was proposed by $\mathrm{Li}$ and Papadopoulos (2002) and involved with the empirical estimator of $g\left(\mu_{1}, \mu_{2}, \mu_{3}\right)$. In the sequel, however, we will modify their test by imposing the property of affine invariance - a desired property for any goodness-of fit test for the Laplace distribution.

More specifically, the proposed test will be based on the transformed data $\hat{Y}_{i}=\left(X_{i}-\hat{\delta}_{n}\right) / \hat{c}_{n}$ and an empirical estimator $T_{n}$ of $g\left(\mu_{1}, \mu_{2}, \mu_{3}\right)$ based on them. The asymptotic distribution of $T_{n}$ is derived under the null hypothesis of Laplace in the following theorem.

Theorem 3.1. Let $\left(X_{1}, X_{2}, \ldots, X_{n}\right)$ be a random sample from $\mathcal{C} L(\delta, c)$ and $\hat{Y}_{i}=\left(X_{i}-\hat{\delta}_{n}\right) / \hat{c}_{n}$, $i=1, \ldots, n$, be the transformed data. Let

$$
T_{n}=\frac{1}{n} \sum_{i=1}^{n} \hat{Y}_{i}^{3}-3 \frac{1}{n^{2}} \sum_{i=1}^{n} \hat{Y}_{i} \sum_{i=1}^{n} \hat{Y}_{i}^{2}+2\left(\frac{1}{n} \sum_{i=1}^{n} \hat{Y}_{i}\right)^{3},
$$

where

$$
\hat{Y}_{i}=\frac{X_{i}-\hat{\delta}_{n}\left(X_{1}, \ldots, X_{n}\right)}{\hat{c}_{n}\left(X_{1}, \ldots, X_{n}\right)},
$$

$\hat{\delta}_{n}=\hat{\delta}_{n}\left(X_{1}, \ldots, X_{n}\right)$ and $\hat{c}_{n}=\hat{c}_{n}\left(X_{1}, \ldots, X_{n}\right)$ are the mle's of the parameters $\delta$ and $c$, respectively. Then the statistic $\sqrt{n} T_{n}$ converges in distribution, as $n \longrightarrow \infty$, to the normal distribution $N(0,504)$.

The proof of the Theorem 3.1 is relegated to Appendix A. The results of Theorem 3.1 can be used to construct a necessary test for testing the hypothesis of Laplace. Indeed by the previous theorem it follows that if the null hypothesis $H_{0}$ is true then it is also true that the statistic $Z_{n}=\sqrt{\frac{n}{504}} T_{n}$ is asymptotically $N(0,1)$. Consequently, in view of Theorem 3.1, $H_{0}$ should be rejected at a significance level $\alpha$ if $\left|Z_{n}\right| \geq z_{\alpha / 2}$, which implies departure from the Laplace distribution. On the contrary, failing to reject the null hypothesis implies that no sufficient information is available for drawing any statistical conclusion on the null hypothesis.

However in order to use the asymptotic critical values one should examine the convergence of the percentiles of $Z_{n}$ to those of the $N(0,1)$ distribution subject to the assumption that the data are stemming from a Laplace distribution. To achieve this, a total of $l=100.000$ samples of different sample sizes $(n=20,30,50,60,70,100,200,500,1000,5000)$ were generated from $\mathcal{C} L(0,1)$. Note that one can confine the study to the case of $\mathcal{C} L(0,1)$ since the asymptotic distribution of $T_{n}$ is independent of the parameters $\delta$ and $c$ and $T_{n}$ is affine invariant. For each sample, the value of $Z_{n}$ was computed. Then, based on all $l$ values of $Z_{n}$, Monte Carlo percentiles were computed and compared with the theoretical limiting percentiles. The simulation was carried out by using $\mathrm{R}$ ( $\mathrm{R}$ Core Team 2020). The $\mathrm{R}$ code used is presented in the Appendix B.

The results are displayed in Table 1. The last row of the table displays the corresponding percentiles of the $N(0,1)$ distribution. The results indicate a slow convergence of the critical values to their limiting values. This suggests that the limiting critical values may not provide a good approximation. Hence, for small and moderate sample sizes one can use a parametric bootstrap for computing the $p$-values instead of using the asymptotic distribution. A Monte Carlo study was carried out on the type I error rates in order to examine the performance of the test based on these latter two options. The empirical type I error rates appear in Table 2. They are computed by the relation

$$
\text { Empirical Type I error rate }=\frac{\text { Number of rejections }}{\text { Number of replications }}
$$

at significance levels $\alpha=0.05$ and 0.1 .

From Table 2 it is clear that for small and moderate sample sizes the use of the parametric bootstrap is recommended. This is not a disadvantage of the test since as Gel (2010) already 
Table 1: Simulated lower and upper critical values of $Z_{n}$ indicated for $\alpha$ and $n$ as shown and 100000 simulated samples from $\mathcal{C} L(0,1)$

\begin{tabular}{|c|cc|cc|cc|}
\hline & \multicolumn{2}{|c|}{$\alpha=0.01$} & \multicolumn{2}{c|}{$\alpha=0.05$} & \multicolumn{2}{c|}{$\alpha=0.1$} \\
\hline $\mathrm{n}$ & $Z_{n}^{l}$ & $Z_{n}^{u}$ & $Z_{n}^{l}$ & $Z_{n}^{u}$ & $Z_{n}^{l}$ & $Z_{n}^{u}$ \\
\hline 20 & -2.49739 & 2.40483 & -1.35659 & 1.32971 & -0.95007 & 0.95038 \\
30 & -2.75772 & 2.74174 & -1.50211 & 1.50553 & -1.08818 & 1.09160 \\
50 & -2.96832 & 2.99391 & -1.65895 & 1.69383 & -1.22410 & 1.23730 \\
60 & -2.98985 & 3.07540 & -1.71866 & 1.72124 & -1.27531 & 1.27553 \\
70 & -3.07916 & 3.09744 & -1.76075 & 1.75607 & -1.30965 & 1.29881 \\
100 & -3.06851 & 3.14049 & -1.82853 & 1.83216 & -1.37432 & 1.37556 \\
200 & -3.03301 & 3.03401 & -1.89422 & 1.91534 & -1.46851 & 1.48683 \\
500 & -2.91472 & 2.88816 & -1.94998 & 1.95911 & -1.56223 & 1.57465 \\
1000 & -2.79985 & 2.83282 & -1.95724 & 1.97529 & -1.59362 & 1.60403 \\
5000 & -2.64775 & 2.66607 & -1.96253 & 1.97677 & -1.63014 & 1.63365 \\
\hline$N(0,1)$ & -2.57583 & 2.57583 & -1.95996 & 1.95996 & -1.64485 & 1.64485 \\
\hline
\end{tabular}

Table 2: Simulated type I rate: percentage of samples with p-value smaller that $\alpha \cdot \mathcal{C} L(0,1)$, number of simulated samples: 10,000, p-value: 1) asymptotic and 2) bootstrap p-value: number of bootstrap samples: 1000

\begin{tabular}{|c|cc|cc|cc|}
\hline & \multicolumn{2}{|c|}{$\alpha=0.01$} & \multicolumn{2}{c|}{$\alpha=0.05$} & \multicolumn{2}{c|}{$\alpha=0.1$} \\
\hline$n$ & asymptotic & bootstrap & asymptotic & bootstrap & asymptotic & bootstrap \\
\hline 20 & 0.01 & 0.0116 & 0.0218 & 0.0508 & 0.0325 & 0.1002 \\
30 & 0.0125 & 0.0096 & 0.0281 & 0.0533 & 0.0443 & 0.0998 \\
50 & 0.0157 & 0.0093 & 0.0307 & 0.0482 & 0.0502 & 0.0944 \\
60 & 0.0172 & 0.0095 & 0.0363 & 0.0494 & 0.0555 & 0.101 \\
70 & 0.0155 & 0.0081 & 0.0346 & 0.0467 & 0.0578 & 0.0947 \\
100 & 0.0185 & 0.01 & 0.0417 & 0.0521 & 0.0695 & 0.1024 \\
\hline
\end{tabular}


pointed out critical values of other available tests detecting departures from the Laplace distribution can be obtained either from special tables or from a Monte Carlo study.

The $\mathrm{R}$ function for the proposed goodness-of-fit test with options for bootstrap $p$-value and $p$-value based on the asymptotic standard normal distribution is displayed in Appendix B. In the simulation study of the next section as well as in practice someone can utilize - taking into account that the test based on $Z_{n}$ is a two-tailed - the 100( $\left.\alpha / 2\right)$-th and 100(1-a/2)-th percentiles of the empirical distribution of the test statistic.

\subsection{Entropy and divergence based tests}

Entropy and divergence based tests are widely common for testing departures from a hypothesized distribution. In this section some existing tests are briefly presented.

\section{Maximum entropy test}

Choi and Kim (2006) presented three goodness-of-fit tests for the Laplace distribution based on its maximum entropy characterization result. However, based on a Monte Carlo study they recommended the use of only one of these tests. Accordingly we briefly discuss in the sequel only this recommended test.

Let $X$ be a r.v. with density function $f_{X}(x)$. The Shannon's entropy (see Shannon (1948)) of $X$ is defined by $H\left(f_{X}\right)=E\left(-\log \left(f_{X}(x)\right)\right)$. Choi and Kim (2006) proved that under the restriction that $E|X|=c$ the distribution of $X$ maximizing the Shannon's entropy is the $\mathcal{C} L(0, c)$ and its entropy is $H\left(f_{X}\right)$. Utilizing this maximum entropy characterization result, Choi and Kim (2006) proposed a test statistic for testing departures from the Laplace distribution based on an estimation of the entropy difference between the data-generating distribution and the hypothetical distribution. In this frame, a parametric procedure for estimating the entropy of the hypothetical distribution and a nonparametric one for estimating the entropy of the data-generating distribution were used. Following this estimation procedure, the entropy based gof test statistic is given by

$$
T_{m, n}^{V}=\frac{n}{2 m \hat{c}_{n}}\left\{\prod_{i=1}^{n}\left(\hat{V}_{(i+m)}-\hat{V}_{(i-m)}\right)\right\},
$$

where $m$, denoting the window size, is a positive integer smaller than $n / 2$ while $\hat{V}_{(i-m)}=\hat{V}_{(1)}$ for $i \leq m$ and $\hat{V}_{(i+m)}=\hat{V}_{(n)}$ for $i \geq n-m$. According to Choi and Kim (2006), $m$ is selected in advance and its optimal choice corresponding to a given sample size $n$ was studied for various sample sizes up to 100. In Table 4 by Choi and Kim (2006) the results of this study is given for $n \leq 50$, while the rest of the results are available upon request from the authors. In the simulation study the values $m=3$ for $n=20, m=6$ for $n=50$ and $m=13$ for $n=100$ were used.

Based on the asymptotic results obtained by Choi and Kim (2006) the null hypothesis of Laplace is rejected when the test statistic is less than the corresponding critical value at a designated significance level $\alpha$ (left-tailed). $T_{m, n}^{V}$ is approximately normally distributed under the null hypothesis. Its asymptotic variance however is not easy to be derived. Consequently, we use the empirical critical values which are the $100 a$-th percentiles of the empirical distribution. For more details on the $T_{m, n}^{V}$ tests and a table of critical values for selected values of $m$ and $n$ see Choi and Kim (2006).

\section{Energy distance test}

The idea behind the class of energy distance goodness-of-fit test is based on the following characterization of equality of distributions: if $X_{1}$ and $Y_{1}$ are independent random variables such that $E\left|X_{1}\right|<\infty$ and $E\left|Y_{1}\right|<\infty$, with cumulative distribution functions $F_{1}$ and $G_{1}$, 
respectively then

$$
D^{2}\left(F_{1}, G_{1}\right)=2 E\left|X_{1}-Y_{1}\right|-\left|X_{1}-X_{1}^{\prime}\right|-\left|Y_{1}-Y_{1}^{\prime}\right| \geq 0
$$

with equality to zero if and only if $X_{1}$ and $Y_{1}$ are identically distributed, where $X_{1}^{\prime}$ and $Y_{1}^{\prime}$ are i.i.d. copies of the random variable $X_{1}$ and $Y_{1}$, respectively; that is, $X_{1}$ and $X_{1}^{\prime}$ are i.i.d., and $Y_{1}$ and $Y_{1}^{\prime}$ are i.i.d. Note that energy distance between the distributions $F_{1}$ and $G_{1}$ is defined to be the square root of $D^{2}\left(F_{1}, G_{1}\right)$.

As pointed out by Rizzo and Szekely (2016), energy distance is a metric that measures the distance between the distributions of random variables or vectors and is zero if and only if the distributions are identical. Thus energy distance characterizes equality of distributions and provides a theoretical foundation for statistical inference and goodness-of-fit tests. For a review and implementation of the energy goodness-of-fit test we refer to Rizzo and Szekely (2016).

In the frame of goodness-of-fit test the distributions to be compared are the hypothesized distribution given in the null hypothesis and the sample distribution. Rizzo and Haman (2016) presented the results related with the expected distance of a random variable $X$ which follows asymmetric Laplace distribution from an arbitrary point and with the expected distance $E\left|X-X^{\prime}\right|$ when $X$ and $X^{\prime}$ are independent and identically asymmetric Laplace distributed. Taking into account that symmetric Laplace distribution is a special case of the asymmetric one and the previous mentioned results, the test statistic proposed by Rizzo and Haman (2016) is given by the following relation:

$$
E_{n}=2 \sum_{i=1}^{n}\left(\left|\hat{Y}_{i}\right|+\exp \left(-\left|\hat{Y}_{i}\right|\right)\right)-1.5 n-\frac{2}{n} \sum_{k=1}^{n}(2 k-1-n) \hat{Y}_{(k)},
$$

where $\hat{Y}_{i}, i=1, \ldots, n$ were defined in Section 2. The null hypothesis of Laplace distribution is rejected for large values of $E_{n^{-}}$implying a right tailed test- the critical values are taken to be the $100(1-\alpha)$-th percentiles of the empirical distribution of $E_{n}$.

\section{Alizadeh Noughabi and Balakrishnan tests}

Divergence measures are indices of similarity or dissimilarity between populations and are used for the development of statistical methods in order to formulate and solve a great variety of statistical problems (see the monograph by Pardo (2006)). One of the widely used and studied divergence measure which includes many others as special cases is the family of divergence measures which is known as $\phi$-divergence and were defined simultaneously by Csiszar (1963) and Ali and Silvey (1966). Let $P$ and $Q$ denote two probability measures over a measurable space $M$ such that $P$ is absolutely continuous with respect to $Q$, then the $\phi$-divergence is defined as

$$
D_{\phi}(P, Q)=\int_{M} \phi\left(\frac{d P}{d Q}\right) d Q
$$

where $\phi:[0,+\infty) \rightarrow(-\infty, \infty)$ is a convex and continuous function such that $\phi(1)=\phi^{\prime}(1)=0$, $0 \phi\left(\frac{0}{0}\right)=0$ and $0 \phi\left(\frac{p}{0}\right)=p \lim _{u \rightarrow+\infty} \frac{\phi(u)}{u}$. Well-known divergence measures are constructed by suitable choices of $\phi$. For instance the Kullback-Leibler (Kullback and Leibler (1951)) is obtained when $\phi(t)=t \log (t)$. Notice that for all probability measures $D_{\phi}(P, Q) \geq 0$, while $D_{\phi}(P, Q)=0$ if $P=Q$.

As a consequence of this last property, a goodness-of-fit test can be constructed based on an estimate of the $\phi$-divergence between the true density of the observations $X_{1}, \ldots, X_{n}$ and the hypothesized distribution under the null hypothesis. Based on this idea Alizadeh Noughabi and Balakrishnan (2016) introduced a general goodness-of-fit test based on an estimate through kernel density estimation of this divergence. In their study Alizadeh Noughabi and Balakrishnan (2016) considered five different choices for the function $\phi$ function and applied them to the goodness-of-fit test for the normal, exponential, uniform and Laplace distributions. 
Based on a Monte Carlo study Alizadeh Noughabi and Balakrishnan (2016) concluded that for the Laplace distribution, the test based on Kullback-Leibler divergence performs quite well as compared to the EDF tests and the other four tests proposed by them by using different member of the $\phi$-divergence. For this reason, we only consider this test in our simulation study which is defined as follows:

$$
T_{K L}=\frac{1}{n} \sum_{i=1}^{n} \log \left(\frac{\hat{f}\left(X_{i}\right)}{f_{0}\left(X_{i} ; \hat{\delta}_{n}, \hat{c}_{n}\right)}\right)
$$

where $f_{0}(x ; \delta, c)$ is the p.d.f. of the classical Laplace distribution $\mathcal{C} L(\delta, c)$ given in $(1)$, while $\hat{f}$ is a kernel density estimator of the unknown true density. Alizadeh Noughabi and Balakrishnan (2016) proposed (see p. 414) to use the following kernel density estimator

$$
\hat{f}\left(X_{i}\right)=\frac{1}{n s 1.06 n^{-1 / 5}} \sum_{j=1}^{n} f\left(\frac{X_{i}-X_{j}}{1.06 S_{n} n^{-1 / 5}}\right),
$$

where $S_{n}$ denotes the sample standard deviation which is obtained from relation (7), while $f$ is the p.d.f. of the standard normal distribution. The null hypothesis of Laplace distribution is rejected for large values of $T_{K L^{-}}$implying a right tailed test- the critical values are taken to be the 100(1- $\alpha)$-th percentiles of the empirical distribution of $T_{K L}$. For more details about this class of tests we refer to Alizadeh Noughabi and Balakrishnan (2016), while for more details about $\phi$-divergence measures see Pardo (2006).

\section{Alizadeh Noughabi test}

Let $X_{1}, \ldots, X_{n}$ be a sample of size $n$ from a population with unknown true density say $g(x)$ and suppose that we interest to test the null hypothesis $H_{0}: g(x)=f(x ; \theta)$, for some $\theta \in \Theta$ with $f(x ; \theta)$ the p.d.f. of a parametric family of distributions. Then, as mentioned in the previous subsection, a goodness-of-fit test can be constructed based on an estimator of a divergence measure between $g(x)$ and $f(x ; \theta)$. Motivated by this idea, Alizadeh Noughabi (2019) proposed recently a general statistic for the goodness-of-fit test of statistical distribution which is constructed based on an estimate of Kullback-Leibler divergence. Recall that Kullback-Leibler is a special case of the $\phi$ divergence given in $(27)$ with $\phi(t)=t$ logt. Thus the Kullback-Leibler between $g(x)$ and $f(x ; \theta)$ is given by:

$$
\begin{aligned}
D_{K L}(g, f) & =\int g(x) \log \left(\frac{g(x)}{f(x ; \theta)}\right) d x \\
& =E_{g}(\log g(X))-E_{g}(\log f(X ; \theta)) \\
& =-H\left(g_{X}\right)-E_{g}(\log f(X ; \theta))
\end{aligned}
$$

where $H\left(g_{X}\right)$ is Shannon's entropy. In this frame, Alizadeh Noughabi (2019) proposed to estimate the entropy term by Vasicek's estimate and the term $E_{g}(\log f(X ; \theta))$ by a semiparametric estimate. In this frame the proposed test statistic for testing departures from Laplace distribution is the following:

$$
D A=-\frac{1}{n} \sum_{i=1}^{n} \log \left(\frac{n}{2 m}\left(Z_{(i+m)}-Z_{(i-m)}\right)\right)
$$

where $m$ denoting the window size is a positive integer smaller than $n / 2$, while $Z_{(i-m)}=Z_{(1)}$ for $i \leq m$ and $Z_{(i+m)}=Z_{(n)}$ for $i \geq n-m$. Alizadeh Noughabi (2019) mentioned that the optimal choice of $m$ equals 4 for $n=10, m=7$ for $n=20, m=15$ for $n=100$ and that it increases with $n$ while the ration $m / n$ tends to zero. In our simulation study the value $m=10$ for $n=50$ was also used. The null hypothesis of Laplace distribution is rejected for large values of $D A$ - implying a right tailed test- the critical values are taken to be the 
$100(1-\alpha)$-th percentiles of the empirical distribution of $D A$. For more details about this test we refer to Alizadeh Noughabi (2019).

\section{Alizadeh Noughabi and Park tests}

Alizadeh Noughabi and Park (2016) proposed several test statistics for testing departures from the Laplace distribution. Their idea is also motivated by the Kullback-Leibler divergence between the unknown true density $g(x)$ and the hypothesized density under the null hypothesis, i.e. the density of the Laplace distribution. From relation (30) it is easily obtained that in the case of Laplace distribution

$$
D_{K L}\left(g, f_{0}\right)=-H\left(g_{X}\right)+\log (2 c) E_{g}(|X-\delta|) .
$$

In this frame, Alizadeh Noughabi and Park (2016) proposed to use the minimum discriminant information loss estimator for the unknown parameters instead of the maximum likelihood (see Alizadeh Noughabi and Park (2016) for further details). On the other hand since the problem of estimation of $H\left(g_{X}\right)$ has been considered by several authors, Alizadeh Noughabi and Park (2016) obtained five different test statistics based on five different estimators of Shannon's entropy and the moments of nonparametric distribution functions of the aforementioned estimators. Based on a Monte Carlo study Alizadeh Noughabi and Park (2016) concluded that the statistic which uses the Vasicek entropy estimator and the minimum discriminant information loss estimator has a good performance against symmetric alternatives and better performance against asymmetric alternatives. According we only consider this last test in our simulation study which is defined as follows:

$$
T V_{m n}=\log \left(2 \hat{c}_{u}\right)+1-H V_{m n},
$$

where

$$
H V_{m n}=\frac{1}{n} \sum_{i=1}^{n} \log \left(\frac{n}{2 m}\left(X_{(i+m)}-X_{(i-m)}\right)\right),
$$

where $m$ denoting the window size is a positive integer smaller than $n / 2$, while $\hat{c}_{u}$ is defined (see Lemma 3 in Alizadeh Noughabi and Park (2016))

$$
\hat{c}_{u}= \begin{cases}-\frac{1}{n} \sum_{i=1}^{n / 2} \frac{\xi_{i}+\xi_{i+1}}{2}+\frac{1}{n} \sum_{i=n / 2+1}^{n} \frac{\xi_{i}+\xi_{i+1}}{2} & \text { if } n \text { is even } \\ -\frac{1}{n} \sum_{i=1}^{(n-1) / 2} \frac{\xi_{i}+\xi_{i+1}}{2}+\frac{\xi_{(n+1) / 2+1}-\xi_{(n+1) / 2}}{4 n}+ & \\ \frac{1}{n} \sum_{i=(n+1) / 2+1}^{n} \frac{\xi_{i}+\xi_{i+1}}{2} & \text { if } n \text { is odd }\end{cases}
$$

where

$$
\xi_{i}=\frac{X_{(i-m)}+\ldots+X_{(i+m-1)}}{2 m}
$$

with $X_{(i-m)}=X_{(1)}$ for $i \leq m$ and $X_{(i+m)}=X_{(n)}$ for $i \geq n-m$. The proposed values of $m$ for different values of sample size $n$ are given in Table 6 by Alizadeh Noughabi and Park (2016). In our simulation study the values $m=4$ for $n=20, m=6$ for $n=50$ and $m=8$ for $n=100$ were used. The null hypothesis of Laplace distribution is rejected for large values of $T V_{m n^{-}}$implying a right tailed test- the critical values are taken to be the $100(1-\alpha)$-th percentiles of the empirical distribution of $T V_{m n}$. For more details about this class of tests we refer to Alizadeh Noughabi and Park (2016).

\subsection{Other tests}

In this section a goodness-of fit test for the Laplace distribution which cannot be classified in either one of the previous classes is briefly presented. 


\section{Gulati test}

It is well known that if $X \sim \mathcal{C} L(\delta, c)$ then $Y=|X-\delta| \sim \operatorname{Exp}(c)$ where $\operatorname{Exp}(c)$ stands for the exponential distribution with mean $c$. Based on this property Gulati (2011) proposed a goodness-of fit test for the Laplace distribution based on the regression test of Brain and Shapiro (1983) for exponentiality. In this frame, let

$$
W_{i}=(n-i+1)\left(\hat{U}_{(i)}-\hat{U}_{(i-1)}\right), i=1, \ldots, n,
$$

with $\hat{U}_{(0)}=0$ and $\hat{U}_{(i)}, i=1, \ldots, n$ as defined in Section 2. Also let $l_{i}=\frac{\sum_{j=1}^{i} W_{j}}{\sum_{j=1}^{n} W_{j}}$, for $i=1, \ldots, n-1$ and $\bar{l}=\frac{\sum_{i=1}^{n-1} l_{i}}{n-1}$. Then the test statistic proposed by Gulati (2011) is defined by

$$
Z=Z_{1}^{2}+Z_{2}^{2}
$$

where

$$
Z_{1}=\sqrt{12(n-1)}(\bar{l}-0.5) \text { and } Z_{2}=\sqrt{\frac{5(n-1)}{(n+1)(n-2)}}\left(n-2+6 n \bar{l}-12 \sum_{i=1}^{n-1} \frac{i l_{i}}{n-1}\right) .
$$

Based on Gulati (2011) under the null hypothesis this test statistic is asymptotically a chisquare variate with 2 degrees of freedom so that the null hypothesis is rejected if $Z \geq \chi_{1-\alpha, 2}^{2}$. Based on a Monte Carlo study Gulati (2011) concluded that the empirical percentiles were fairly close to the theoretical percentiles of the chi-square distribution. Since the test is righttailed we use in our simulation section the empirical critical values which are the $100(1-\alpha)$-th percentiles of the empirical distribution. For more details on $Z$ test see Gulati (2011).

\section{Monte Carlo study}

Our purpose is to present a detailed comparison of the existing procedures for departure from the Laplace distribution. To assess the performance of the above tests we apply them to alternatives distributions (symmetric and asymmetric) which were previously considered in other studies of testing departures from the Laplace distribution (c.f. Puig and Stephens 2000b; Best et al. 2008; González-Estrada and Villaseñor 2016). In this context, the performance of the power of all the tests is investigated using Monte Carlo simulations by generating samples for the following alternatives:

- Symmetric alternatives: normal $N(0,1)$, Logistic $L(0,0.551)$, Cauchy, Uniform $(-1.732$, 1.732), $\operatorname{Beta}(2,2), t$ with 10,6 and 3 degrees of freedom (d.f.'s), Tukey, contaminated normal $C N_{3.2,0.2}$ and $C N_{3.5,0.1}$, two special cases of the normal inverse Gaussian (NIG) distribution denoted by $N I G_{1}$ and $N I G_{2}$ by Gel (2010), i.e., these are NIG distributions with common skewness and location parameters 0 and 1 , respectively, but with a combination of shape and scale parameters $(0.4,0.6)$ and $(0.7,0.2)$, respectively.

- Asymmetric alternatives: $\operatorname{Exp}(1), \operatorname{Gamma}(2,1)$, standard Gumbel, skew-normal with slant parameter 3, skew-t with slant parameter 3 and 10 d.f., Log-Normal, Weibull $(2,1)$, Weibull $(3,1), \chi^{2}$ with 2 degrees of freedom (d.f.'s), Extreme Value $(0,1)$, Inverse Gaussian IG(4), two special cases of Normal Inverse Gaussian distribution denoted by $N I G_{3}$ and $N I G_{4}$ by Gel (2010), i.e., NIG distributions with common location parameter 1 , but with a combination of shape, skewness and scale parameters $(1,0.5,0.43)$ and $(0.5,0.2,0.5)$, respectively.

Only the exact simulated critical values are utilized for the power analysis in order to ensure that the correct size of the test is preserved. For this purpose we initially use the many.crit 
function of the PoweR package (see Lafaye de Micheaux and Tran (2016)) for the determination of the critical values based on $l=100,000$ samples from $\mathcal{C} L(0,1)$. Then by employing the function powcomp. fast of the PoweR package (see Lafaye de Micheaux and Tran (2016)), the empirical power of the tests are obtained by using the above set of critical values. Note that for implementing the existing gof tests for the Laplace distribution we have used the functions of the PoweR package with the exception for the $K$ test by Gel (2010), the two tests $R_{n}$ and $R_{n}^{\prime}$ proposed by González-Estrada and Villaseñor (2016), the modified test based on moment structure studied in this paper in details and the tests presented in the subsection 3.4, i.e. the entropy and divergence based tests, denoted by $T_{m, n}^{V}, E_{n}, T_{K L}, D A$ and $T V_{m n}$, proposed by Choi and Kim (2006) Rizzo and Haman (2016), Alizadeh Noughabi and Balakrishnan (2016), Alizadeh Noughabi (2019) and Alizadeh Noughabi and Park (2016), respectively. The empirical power is then obtained by calculating the proportion of times in $l=100,000$ Monte Carlo simulations for which the false null hypothesis is rejected. For all of the latter we take into account the specified significance level for which $l=100,000$ samples of size $n$ $(n=20,50,100)$ are simulated from the previous distributions. A relevant $\mathrm{R}$ code is presented in the Appendix.

Based on the results given in Tables 3 and 4 we conclude the following:

a) For symmetric alternatives it is observed that $R_{n}$ and $R_{n}^{\prime}$ proposed by González-Estrada and Villaseñor (2016) produce similar results, while $R_{n}$ outperforms $R_{n}^{\prime}$ in the majority of the asymmetric alternatives. Accordingly, the rest of the conclusions is restricted to $R_{n}$ only. Despite the fact that the $R_{n}$ test is very simple and is based on a ratio of two estimators for the scale parameter of the Laplace distribution, it is found to be rather competitive for the symmetric alternatives.

b) Between the five e.d.f. gof tests, i.e., $W^{2}, U^{2}, A^{2}, \sqrt{n} D$ and $V$, the Watson $\left(U^{2}\right)$ is found to be the best versus symmetric alternatives. For non-symmetric alternatives the Anderson-Darling $\left(A^{2}\right)$ is found to be the best (in 10 out 14 alternatives), though the $U^{2}$ is rather good and the best for 4 out 14 non-symmetric alternatives. These conclusions coincide with that reached by Puig and Stephens (2000a) and with that by Choi and Kim (2006) based on simulation studies with lesser alternatives distributions and simulation runs. Consequently, for the rest of our conclusions, the $U^{2}$ and $A^{2}$ are reported, respectively, for symmetric and asymmetric alternatives.

c) When comparing the e.c.f. tests proposed by Meintanis (2005) it is concluded that for the symmetric alternatives considered with population kurtosis less or equal to 6 , i.e., for the alternatives $U(0,1)$, Tukey $(0.5)$, Beta $(2,2)$, Normal, t with 10 d.f., Logistic, t with 6 d.f., $T_{n, 0.5}^{(2, M L)}$ has the best power. On the other hand for the symmetric alternatives considered with population kurtosis greater than 6 (see $C N_{3.2,0.2}, C N_{3.5,0.1}, N I G_{1}$ and $N I G_{2}$ ) or with undefined or non finite population kurtosis (see Cauchy and t with 3 d.f.), it seems that $T_{n, 2}^{(1, M O)}$ is a good choice among them. Finally, the $M O$-based tests are slightly more powerful for all asymmetric alternatives considered with the exception of the Weibull. The performance of $T_{n, 2}^{(1, M O)}$ and $T_{n, 0.5}^{(2, M O)}$ is almost similar. Consequently, we restrict the rest of the analysis to $T_{n, 0.5}^{(2, M L)}$ for symmetric alternatives with population kurtosis less or equal to 6 and to $T_{n, 2}^{(1, M O)}$ for any other alternatives.

d) When comparing for symmetric alternatives the performance of e.c.f. based test previously recommended with the Watson e.d.f. test, we conclude that e.c.f. based tests outperforms $U^{2}$ (with the exception when testing against the Cauchy and the contaminated normal distributions). For asymmetric alternatives, the performance of $T_{n, 2}^{(1, M O)}$ is better in almost all cases than the Anderson Darling test. Thus in a manner similar to that in Meintanis (2005) we conclude that for the majority of the cases considered the e.c.f. based tests either outperform or remain competitive with the best e.d.f. test. 


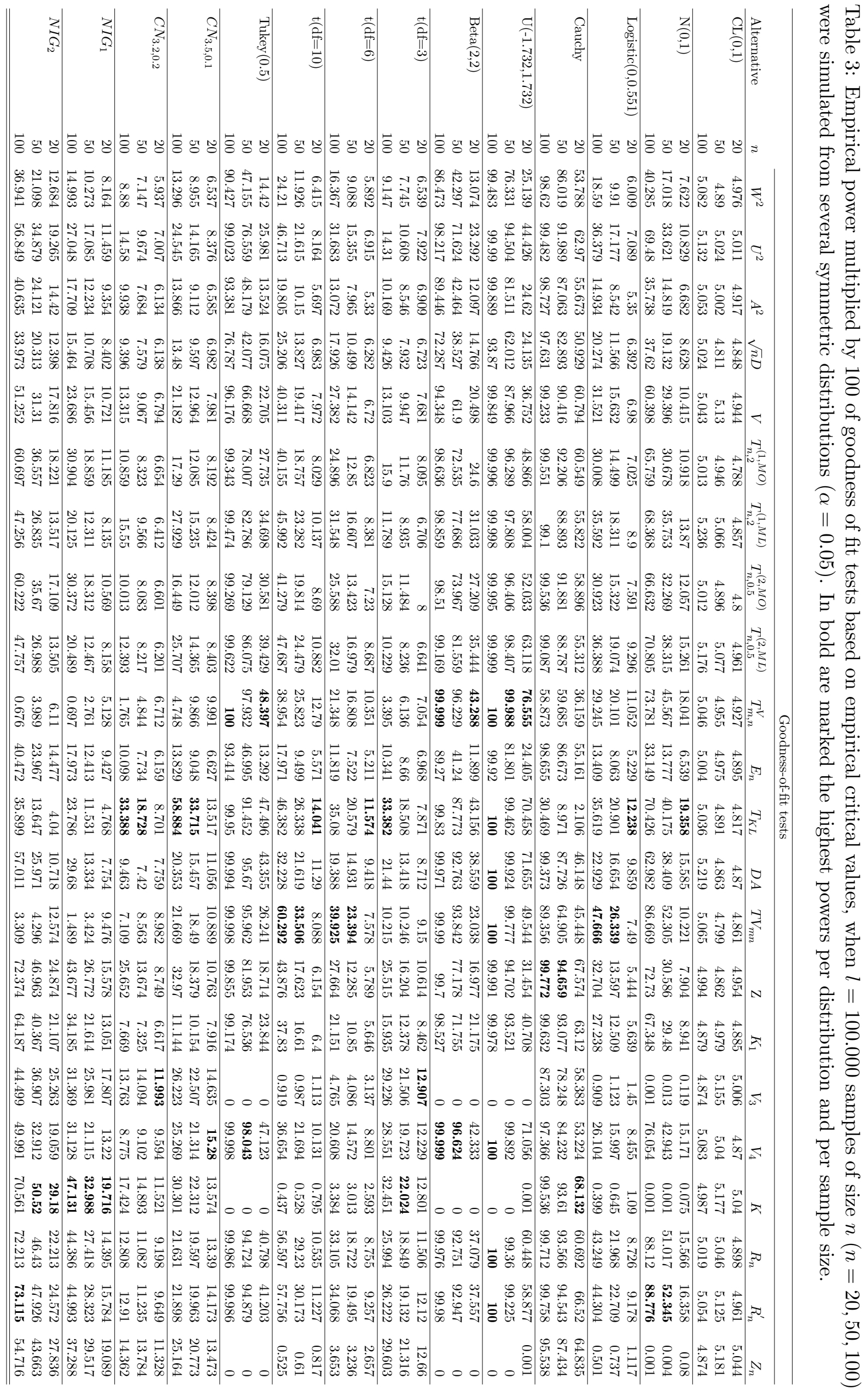




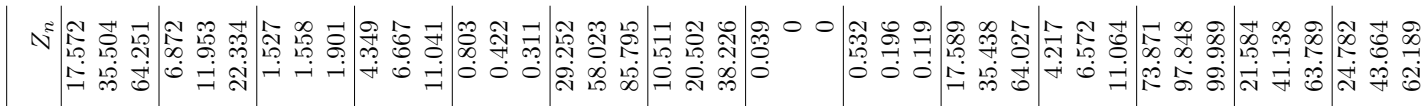

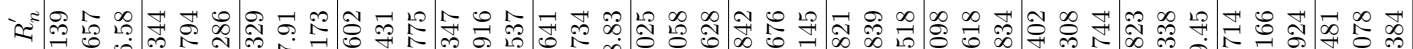

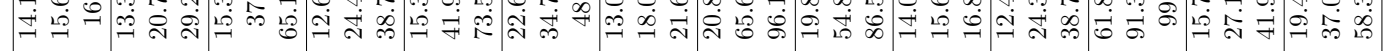

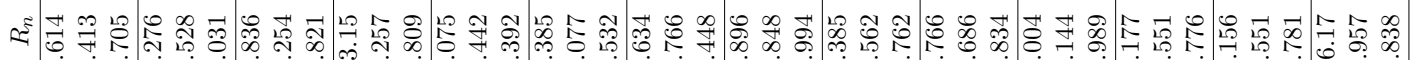

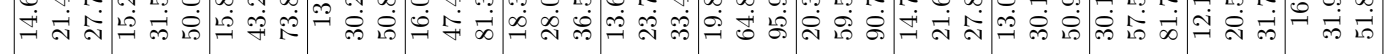

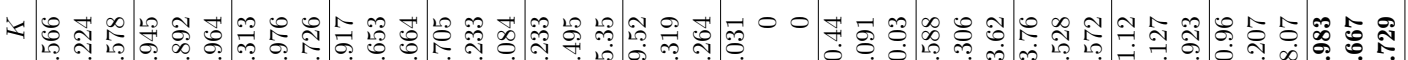

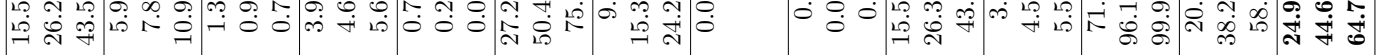

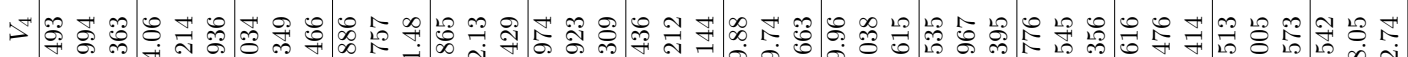
茾隶

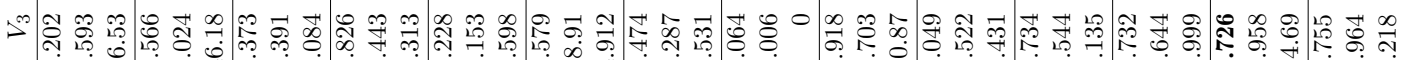

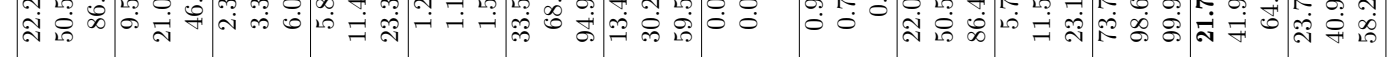

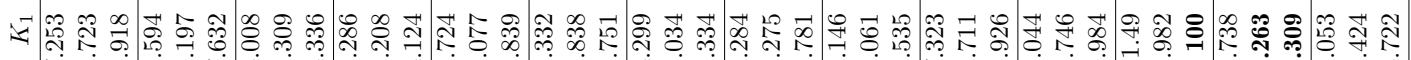
宁

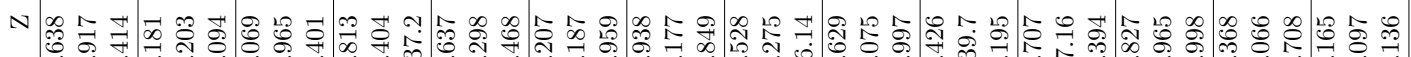

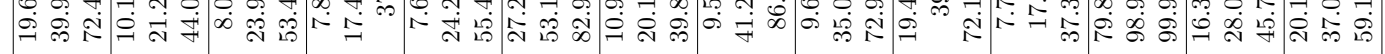

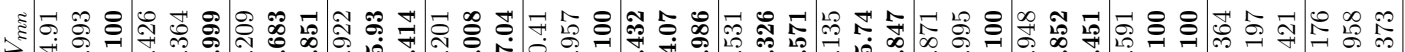

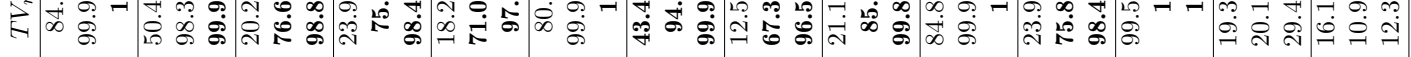

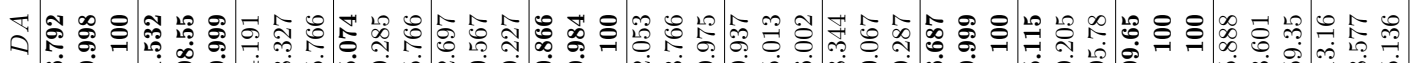
这

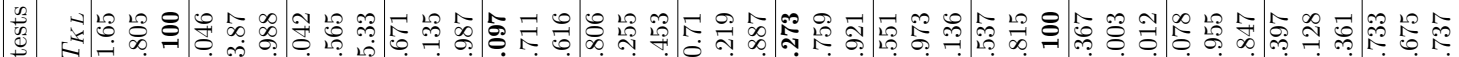

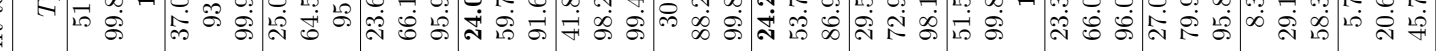

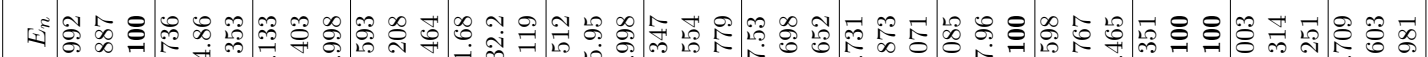

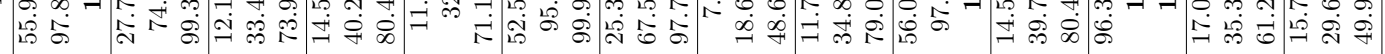

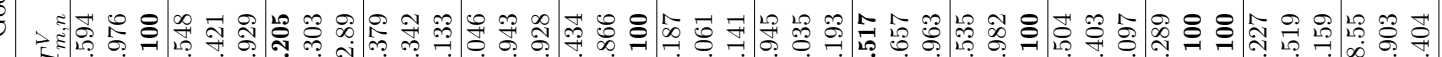

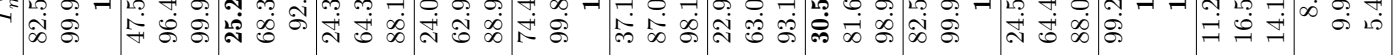

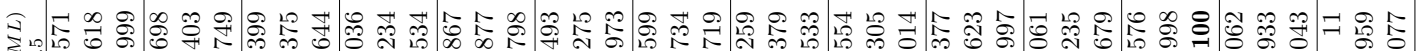

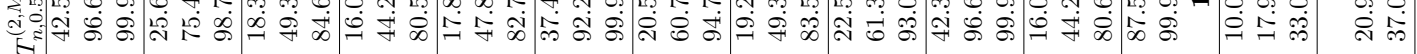

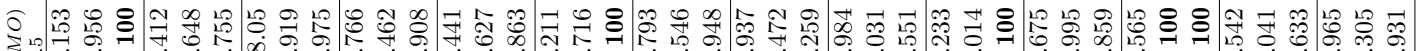

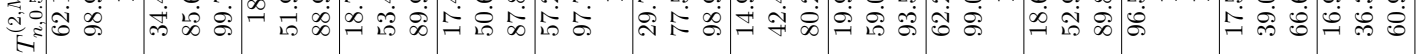

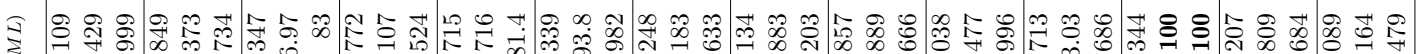

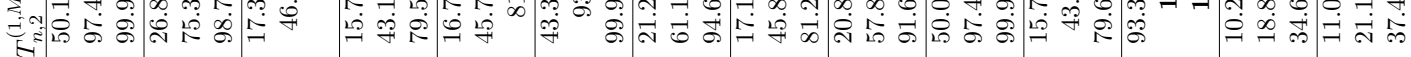

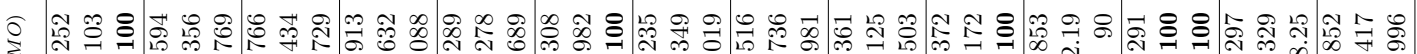

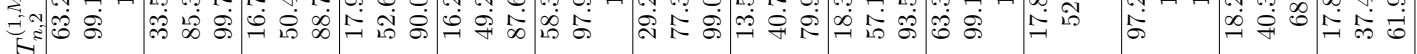

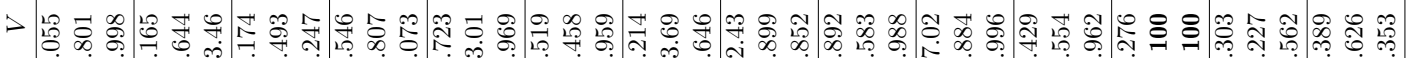

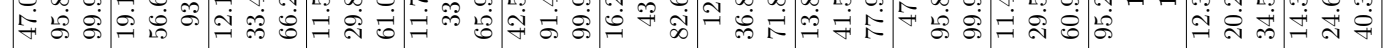

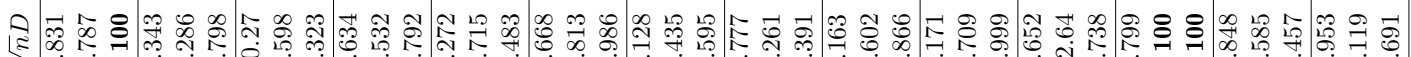

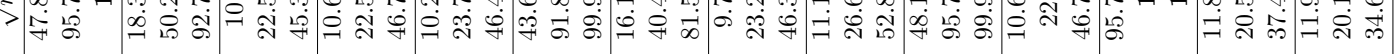

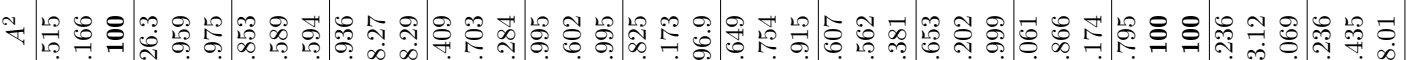

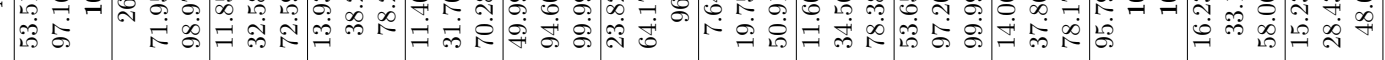

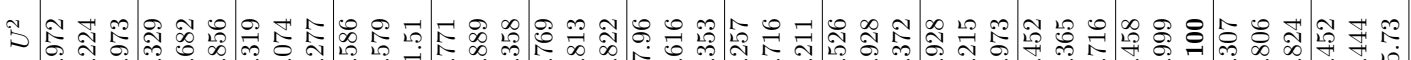

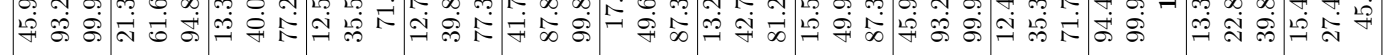

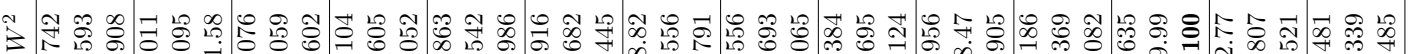

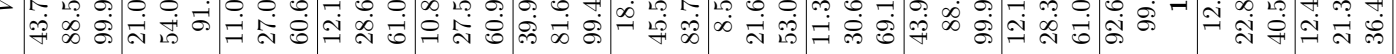

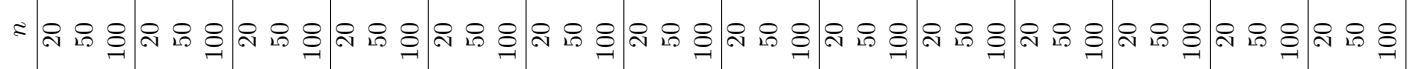
苞 死

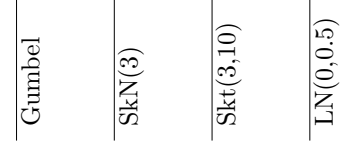

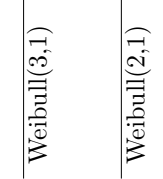


e) For all the alternatives considered $E_{n}$ has less power than the respective of the e.d.f. and e.c.f. based tests previously recommended. Thus this test cannot be recommended. Accordingly the rest of the conclusions is restricted to the rest of the tests belonging to the class of entropy and divergence based tests.

f) When comparing the rest of the entropy and divergence based tests with the e.d.f. and e.c.f. tests previously recommended it is concluded that for symmetric alternatives $T_{m, n}^{V}$ performs better than them under symmetric alternatives with population kurtosis less than or equal to 6 . For this type of alternatives $T_{K L}, D A$ and $T V_{m n}$ behave also similar in almost all cases in comparison with the recommended e.d.f and e.c.f. tests. It seems that for such alternatives $T V_{m n}$ and $T_{m, n}^{V}$ stand out as the best ones, for moderate and large sample sizes, i.e. for $n=50,100$, while for $n=20 T_{m, n}^{V}$ and $T_{K L}$ are good choices among the entropy and divergence based tests. For the rest of the symmetric alternatives we have that the entropy and divergence based tests have less power than the respective of the e.d.f. and e.c.f. previously recommended for the $N I G_{1}, N I G_{2}$ and Cauchy distributions, with $D A$ being the best among them. On the other hand $T_{K L}$ outperforms the e.d.f. and e.c.f. tests previously recommended for contaminated normal and t with 3 d.f. Moreover, for asymmetric alternatives the entropy and divergence based tests have in almost all cases better performance than the recommended e.c.f. and e.d.f. tests with the exception of the NIG distributions. Note that for such alternatives these tests, namely $T_{m, n}^{V}, T_{K L}, T V_{m n}$ and $D A$, have the better performance among the tests considered. Specifically, for asymmetric alternatives with the exception of NIG distributions, $T V_{m n}$ is recommended for moderate and large sample sizes for, while $D A$ is recommended for $n=20$.

However at this point we have to note that there are two points that make the use of the four previously mentioned entropy and divergence based tests difficult or even impossible in some cases in practical applications. The first point is related to the fact that $T_{m, n}^{V}$, $D A$ and $T V_{m n}$ depend on the optimal choice of window sizes $m$. These choices are available for the $T V_{m n}$ for several sample sizes in Table 6 by Alizadeh Noughabi and Park (2016), while for the other two tests are available for specific choices of $n$. Nonetheless, this problem can be tackled by noticing, based on the available information, that for $T_{m, n}^{V}$ it seems that the optimal choice of $m$ is about $13 \%-15 \%$ of the size of $n$. On the other hand, $D A$ was constructed based on specific choice of kernel density estimator. The second point is that for instance $T_{m, n}^{V}$ is based on the geometric mean of a suitable transformation of the data which involves differences related to their median, while the rest of the tests involve the logarithm of some differences. As a result, whenever we tackle with data points having identical numerical values with that of the median or with differences which are equal to zero, the tests cannot be executed and are not recommended. Note that such a situation is not rare in real data sets due to the rounding up to a certain number of decimal points.

g) As previously noted, the main idea behind the $Z_{n}$ test is that for the Laplace distribution the third central moment equals zero which implies that the population skewness is also zero. This means that its difference with the test based on sample skewness proposed by Rayner and Best (1989), denoted by $V_{3}$, is that only the numerator is used and this does not estimate the standard deviation as $V_{3}$ does. The $Z_{n}$ test has similar or superior performance against some symmetrical distributions (Cauchy, $N I G_{i}, i=1,2$ distributions) since estimating the standard deviation in such cases add an extra layer of doubt in the estimation. On the other hand the $Z_{n}$ test has inferior or similar performance for non symmetric alternatives since the presence of an estimator of the standard deviation allows the existence of a skewed distribution to be more easily spotted.

h) The $K$ test by Gel (2010) which is based on a combination of the sample skewness and kurtosis; the smooth $V_{3}$ test by Rayner and Best (1989) which is related to sample 
skewness; and the modified moment based gof $Z_{n}$ test do not perform well and are not competitive under symmetric alternatives with population kurtosis less than or equal to 6 . On the other hand these tests are competitive for symmetric alternatives such as the Cauchy, t with 3 d.f.'s, $N I G_{1}, N I G_{2}$ and contaminated normal distributions, where in some cases they attain the maximum power. Note also that these tests outperforms the popular edf tests for t with 3 d.f.'s, contaminated normal and normal inverse Gaussian distributions. Furthermore, they do not perform well and are not competitive in comparison with the popular edf tests under the asymmetric alternative distributions that are considered (with the exception of the asymmetric normal inverse Gaussian distribution). For the latter alternative distribution their performance is found to be the best among all tests considered and thus is recommended for possible applications. Note that for the asymmetric alternatives considered, $V_{3}$ is better than $Z_{n}$ which is better than $K$ with the exception of the $N I G_{4}$ distribution.

i) Based on the results given in Table 3, we note that for symmetric alternatives, with the exception of uniform distribution, the $Z$ test by Gulati (2011) outperforms the $K_{1}$ test by Langholz and Kronmal (1991). However, based on the results of Table 4, the situation is reversed in favor of the $K_{1}$ test. Moreover, the smooth test $V_{4}$ by Rayner and Best (1989) which is related to sample kurtosis is better than the $Z$ and the $K_{1}$ tests for symmetric alternatives with the exception of the Cauchy and the two normal inverse Gaussian distributions.

Based on the previous results and comments it is concluded that for the majority of the considered asymmetric alternatives there is a power advantage in using the $D A$ and $T V_{m n}$ tests, while for symmetric alternatives a single recommendation is not that clear, since, as expected, there is no test that can detect all types of symmetric alternatives. There are several tests including $T V_{m n}, T_{K L}, T V_{m n}, Z, V_{4}$ and $K$ that present the highest power under different alternatives. Although, it is for note, that $T V_{m n}$ present a robust, acceptable behavior under the symmetric alternatives used with tails not heavier than that of the standard Laplace distribution. On the other hand, despite the fact that in general some tests, for instance the $Z_{n}$ or $V_{3}$, are not useful for detecting several alternatives, they are quite competitive when alternatives with tails heavier than that of the standard Laplace distribution as the Cauchy, $\mathrm{t}$ with 3 d.f.'s, contaminated normals and normal inverse Gaussian distributions are considered. These tests may not present the highest power but have a competitive behavior to that of the tests with the highest power under such alternatives. The same holds for these two tests and for asymmetric alternatives with heavy tailness of large degree. Finally, for symmetric alternatives the simple $R_{n}$ test could be also recommended.

\section{Conclusions}

The main contribution of the paper is linked to the fact that twenty two different goodness-offit tests for the Laplace distribution, included a new one, have been compared with 27 possible alternative distributions (symmetric and asymmetric). As has been initially expected there is no test which outperforms the others in all cases. However if a particular alternative is suspected like the ones mentioned in the simulation study of the previous section, one can utilize the recommended test based on the conclusions of the simulation study. Moreover, extremely practical and important $\mathrm{R}$ codes are provided in the appendix for implementing the comparison study of the various tests for detecting a departure from the Laplace distribution while taking into account a numerous alternative distributions.

\section{Acknowledgements}

The authors would like to thank the anonymous referee for critically reading the manuscript, 
suggesting substantial improvements, such as the inclusion of some competitive tests.

\section{References}

Ali SM, Silvey SD (1966). "A General Class of Coefficients of Divergence of One Distribution from Another." Journal of the Royal Statistical Society. Series B (Methodological), 28(1), $131-142$.

Alizadeh Noughabi H (2019). "A New Estimator of Kullback-Leibler Information and its Application in Goodness of Fit Tests." Journal of Statistical Computation and Simulation, 89(10), 1914-1934.

Alizadeh Noughabi H, Balakrishnan N (2016). "Tests of Goodness of Fit Based on PhiDivergence." Journal of Applied Statistics, 43(3), 412-429.

Alizadeh Noughabi H, Park S (2016). "Tests of Fit for the Laplace Distribution Based on Correcting Moments of Entropy Estimators." Journal of Statistical Computation and Simulation, 86(11), 2165-2181.

Batsidis A, Zografos K (2013). "A Necessary Test of Fit of Specific Elliptical Distributions Based on an Estimator of Song's Measure." Journal of Multivariate Analysis, 113, 91 - 105.

Best DJ, Rayner JCW, Thas O (2008). "Comparison of Some Tests of Fit for the Laplace Distribution." Comput. Stat. Data Anal., 52(12), 5338-5343.

Brain CW, Shapiro SS (1983). "A Regression Test for Exponentiality: Censored and Complete Samples." Technometrics, 25(1), 69-76.

Chen C (2002). "Tests for the Goodness-of-Fit of the Laplace Distribution." Communications in Statistics - Simulation and Computation, 31(1), 159-174.

Choi B, Kim K (2006). "Testing Goodness-of-Fit for Laplace Distribution Based on Maximum Entropy." Statistics, 40(6), 517-531.

Cordeiro GM, Lemonte AJ (2011). "The Beta Laplace Distribution." Statistics and Probability Letters, 81(8), $973-982$.

Csiszar I (1963). "Eine Informationstheoretische Ungleichung und Ihre Anwendung auf den Beweis der Ergodizitat von Markoffschen Ketten." Publications of the Mathematical Institute of the Hungarian Academy of Sciences, 8, 85-108.

Fang KT, Zhu LX, Bentler PM (1993). "A Necessary Test of Goodness of Fit for Sphericity." Journal of Multivariate Analysis, 45(1), 34 - 55.

Fellner WJ (1974). "A Strange Expansion." American Economic Review, 64(2), 71-74.

Gel YR (2010). "Test of Fit for a Laplace Distribution Against Heavier Tailed Alternatives." Computational Statistics $\&$ Data Analysis, 54(4), 958 - 965.

González-Estrada E, Villaseñor JA (2016). "A Ratio Goodness-of-Fit Test for the Laplace Distribution." Statistics \& Probability Letters, 119(C), 30-35.

Gulati S (2011). "Goodness of Fit Test for the Rayleigh and the Laplace Distributions." International Journal of Applied Mathematics and Statistics, 24, 74-85.

Johnson NL, Kotz S, Balakrishnan N (1995). Continuous Univariate Distributions. Number v. 2 in Wiley series in probability and mathematical statistics: Applied probability and statistics. Wiley \& Sons. 
Kotz S, Kozubowski T, Podgorski K (2001). The Laplace Distribution and Generalizations: A Revisit with Applications to Communications, Economics, Engineering, and Finance. Progress in Mathematics. Birkhäuser Boston.

Kozubowski TJ, Nadarajah S (2010). "Multitude of Laplace Distributions." Stat Papers, 127.

Kullback S, Leibler R (1951). "On Information and Sufficiency." Ann. Math. Statistics, 22, 79-86.

Lafaye de Micheaux P, Tran V (2016). "PoweR: A Reproducible Research Tool to Ease Monte Carlo Power Simulation Studies for Goodness-of-Fit Tests in R." Journal of Statistical Software, Articles, 69(3).

Langholz B, Kronmal R (1991). "Tests of Distributional Hypotheses with Nuisance Parameters Using Fourier Series Methods." Journal of the American Statistical Association, 86, 10771084 .

Laplace PS (1774). "Mémoire sur la Probabilité des Causes par les Événements." Mémoires de Mathématique et de Physique, 6, 621-656.

Li G, Papadopoulos A (2002). "A Note on Goodness of Fit Test Using Moments." Statistica, 62, 71-86.

Liang J, Fang K, Hickernell FJ (2008). "Some Necessary Uniform Tests for Spherical Symmetry." Ann Inst Stat Math, 60, 679-696.

Meintanis SG (2005). "A Class of Omnibus Tests for the Laplace Distribution Based on the Empirical Characteristic Function." Communications in Statistics - Theory and Methods, 33(4), 925-948.

Pardo L (2006). Statistical Inference Based on Divergence Measures. Chapman and Hall.

Puig P, Stephens MA (2000a). "Tests of Fit for the Laplace Distribution, with Applications." Technometrics, 42(4), 417-424.

Puig P, Stephens MA (2000b). "Tests of Fit for the Laplace Distribution, with Applications." Technometrics, 42(4), 417-424.

R Core Team (2020). R: A Language and Environment for Statistical Computing. R Foundation for Statistical Computing, Vienna, Austria. URL http://www.R-project.org/.

Rayner JCW, Best DJ (1989). Smooth Tests of Goodness of Fit. Oxford Statistical Science Series. Oxford University Press.

Rizzo ML, Haman JT (2016). "Expected Distances and Goodness-of-fit for the Asymmetric Laplace Distribution." Statistics $\&$ Probability Letters, 117, 158 - 164.

Rizzo ML, Szekely GJ (2016). "Energy Distance." WIREs Computational Statistics, 8(1), $27-38$.

Rublík F (1997). "A Quantile Goodness-of-Fit Test Applicable to Distributions with NonDifferentiable Densities." Kybernetika, 33(5), 505-524.

Shannon CE (1948). "A Mathematical Theory of Communication." Bell System Technical Journal, 27, 379-423, 623-656.

Yen V, Moore A (1988). "Modified Goodness-of-Fit Test for the Laplace Distribution." Communications in Statistics - Simulation and Computation, 17(1), 275-281. 


\section{Appendices}

\section{Appendix A}

Proof of Theorem 3.1. Consider $T_{n}$ as a function of $\hat{\theta}_{n}$ and expand it into a Taylor series around the true value of $\theta=(\delta, c)^{T}$, where $T$ denotes the transpose of a vector or matrix. We will obtain that

$$
\sqrt{n} T_{n}=\sqrt{n} \mathcal{T}_{n}+\left.\sqrt{n}\left(\frac{\partial \mathcal{T}_{n}}{\partial \delta}, \frac{\partial \mathcal{T}_{n}}{\partial c}\right)\right|_{\theta=\theta_{n}^{\star},}\left(\hat{\theta}_{n}-\theta\right),
$$

where

$$
\mathcal{T}_{n}=\frac{1}{n} \sum_{i=1}^{n} Y_{i}^{3}-3 \frac{1}{n^{2}} \sum_{i=1}^{n} Y_{i} \sum_{i=1}^{n} Y_{i}^{2}+2\left(\frac{1}{n} \sum_{i=1}^{n} Y_{i}\right)^{3}
$$

$Y_{i}=\frac{X_{i}-\delta}{c}$ and $\theta_{n}^{\star}=a \hat{\theta}_{n}+(1-a) \theta$, for some $a \in(0,1)$ (note that $\left.\theta_{n}^{\star} \stackrel{\mathrm{P}}{\rightarrow} \theta\right)$.

We first determine the asymptotic joint distribution of $\frac{1}{n} \sum_{i=1}^{n} Y_{i}, \frac{1}{n} \sum_{i=1}^{n} Y_{i}^{2}$ and $\frac{1}{n} \sum_{i=1}^{n} Y_{i}^{3}$, under the hypothesis that $X_{i} \sim \mathcal{C} L(\delta, c), i=1, \ldots, n$, or equivalently, that $Y_{i} \sim \mathcal{C} L(0,1), i=1, \ldots, n$. From the multivariate Central Limit Theorem we easily obtain that under the null hypothesis of Laplace distribution

$$
\sqrt{n}\left(\begin{array}{c}
\frac{1}{n} \sum_{i=1}^{n} Y_{i}-0 \\
\frac{1}{n} \sum_{i=1}^{n} Y_{i}^{2}-2 \\
\frac{1}{n} \sum_{i=1}^{n} Y_{i}^{3}-0
\end{array}\right) \stackrel{\mathrm{L}}{\rightarrow} N_{3}\left(0_{3}, \Sigma\right),
$$

where $0_{3}$ is a 3 -dimensional column vector with zero elements and $\Sigma=\left(\sigma_{l k}\right)_{3 \times 3}$ with $\sigma_{l k}=$ $E Y^{k+l}-E Y^{l} E Y^{k}$. As under the null hypothesis $E Y^{r}=0$ if $r$ is odd and $E Y^{r}=r$ ! if $r$ is even, we obtain that

$$
\Sigma=\left(\begin{array}{ccc}
2 & 0 & 24 \\
0 & 20 & 0 \\
24 & 0 & 720
\end{array}\right)
$$

In order to find the asymptotic distribution of $\mathcal{T}_{n}$ we note that

$$
\mathcal{T}_{n}=g\left(\frac{1}{n} \sum_{i=1}^{n} Y_{i}, \frac{1}{n} \sum_{i=1}^{n} Y_{i}^{2}, \frac{1}{n} \sum_{i=1}^{n} Y_{i}^{3}\right),
$$

where $g(x, y, z)=z-3 x y+2 x^{3}$ and $\dot{g}(x, y, z)=\left(\frac{\partial}{\partial x} g, \frac{\partial}{\partial y} g, \frac{\partial}{\partial z} g\right)=\left(-3 y+6 x^{2},-3 x, 1\right)$. Then by the Cramér's Theorem (see Theorem 7, p. 45, in Ferguson, 1996), we find that under $H_{0}$

$$
\sqrt{n}\left(\mathcal{T}_{n}-g(0,2,0)\right) \stackrel{\mathrm{L}}{\rightarrow} N\left(0, \sigma^{2}\right),
$$

where

$$
\sigma^{2}=\dot{g}(0,2,0) \Sigma \dot{g}^{T}(0,2,0) .
$$

As $g(0,2,0)=0$ and $\dot{g}(0,2,0)=(-6,0,1)$ it follows that $\sigma^{2}=504$. Therefore, under the null hypothesis, the first term $\sqrt{n} \mathcal{T}_{n}$ in the right side of (39) converges in distribution to $N(0,504)$.

For the second term we first note that the quantity $\sqrt{n}\left(\hat{\theta}_{n}-\theta\right)$ is bounded in probability. Then by evaluating the derivative of $\mathcal{T}_{n}$ given in (40) with respect to $\theta$ at the point $\theta=\theta_{n}^{\star}$ it follows that

$$
\frac{\partial \mathcal{T}_{n}}{\partial \delta}=0
$$


and

$$
\frac{\partial \mathcal{T}_{n}}{\partial c}=-\frac{3}{n c^{3}} \sum_{i=1}^{n} Y_{i}+\frac{9}{n^{2} c} \sum_{i=1}^{n} Y_{i} \sum_{i=1}^{n} Y_{i}^{2}-\frac{3}{n^{3} c} \sum_{i=1}^{n} Y_{i}^{3} .
$$

Since under the null hypothesis $X_{i} \sim \mathcal{C} L(\delta, c)$ one obtains that

$$
\left.\frac{\partial \mathcal{T}_{n}(Y)}{\partial \theta}\right|_{\theta=\theta_{n}^{\star}} \stackrel{\mathrm{P}}{\rightarrow} 0 \text { as } n \rightarrow \infty,
$$

and thus the second term on the right side of (39) converges in probability to zero. This completes the proof.

\section{Appendix B}

Next the $R$ code used to obtain the results appearing in the Tables is presented. Note that the following versions of $\mathrm{R}$ packages were loaded during the preparation of the revised version of the current paper:

- PoweR, version 1.0.7 published on August 28, 2018

- univariateML, version 1.1.0 published on August 5, 2020

- MuMIn, version 1.43.17 published on April 15, 2020

- psych, version 1.0.7 published on December 16, 2020

- skewt, version 0.1 published on October 10, 2012

- statmod, version 1.4.35 published on October 19, 2020

- teachingApps, version 1.0.8 published on May 13, 2020

- rmutil, version 1.1.5 published on June 9, 2020

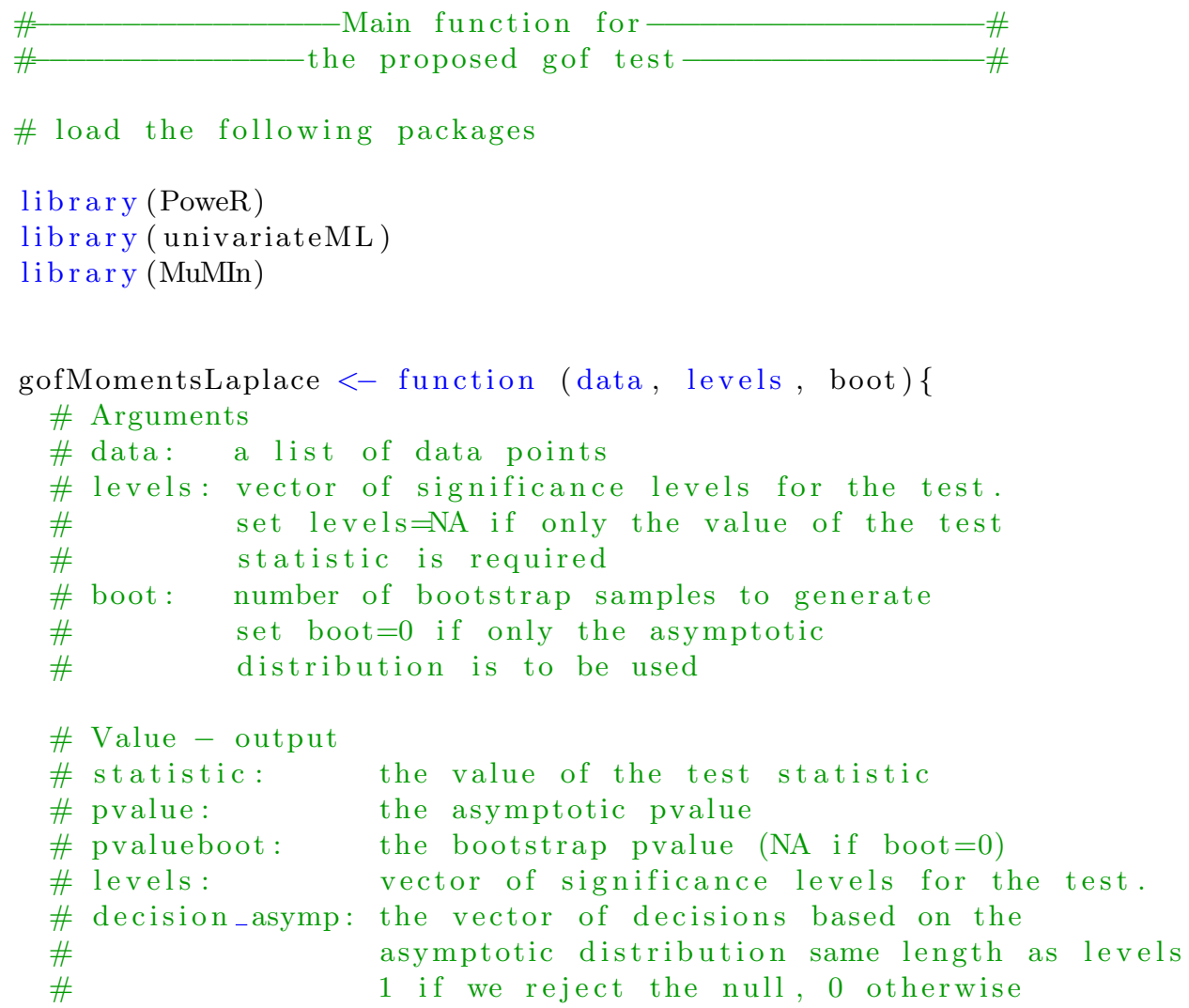




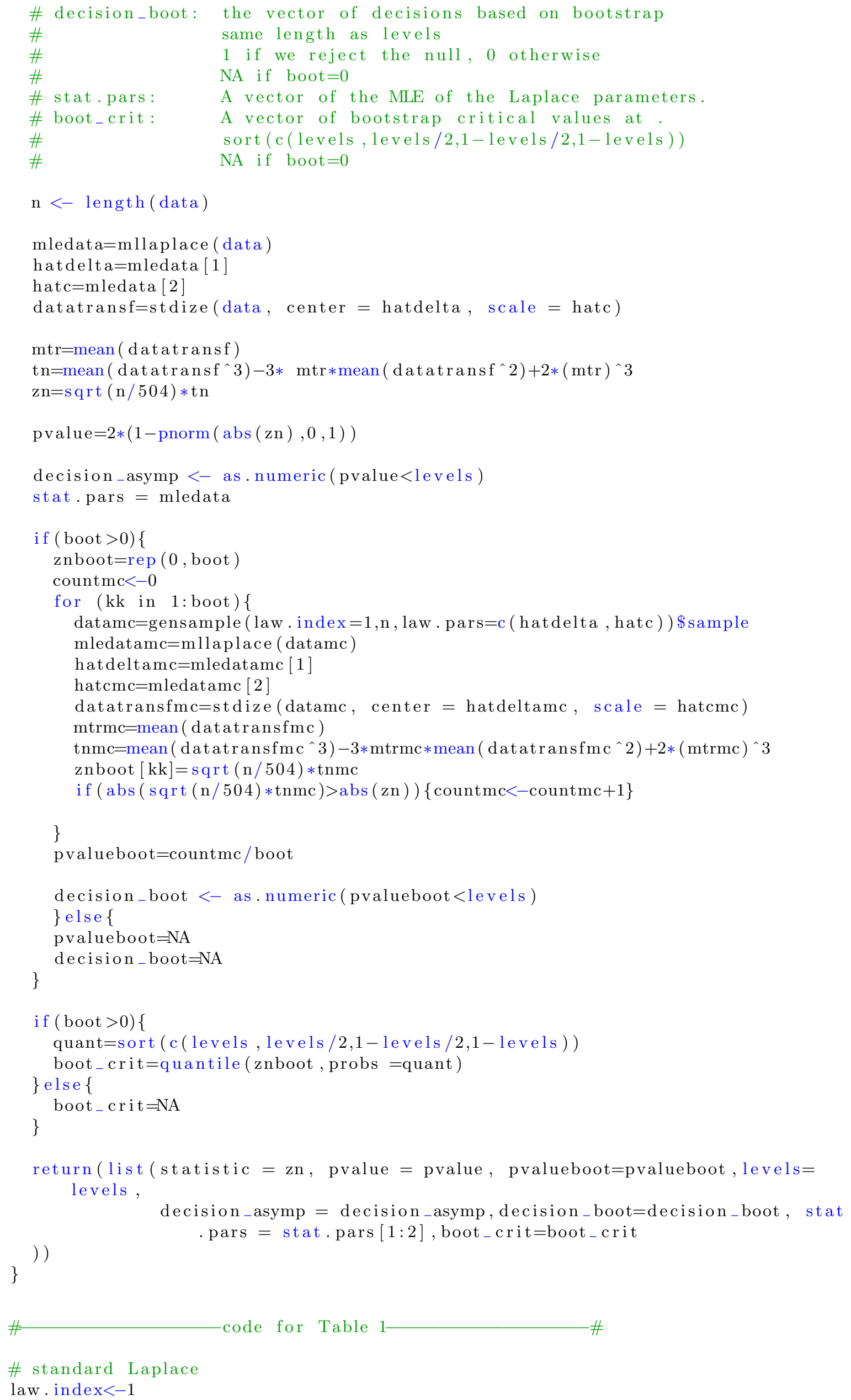




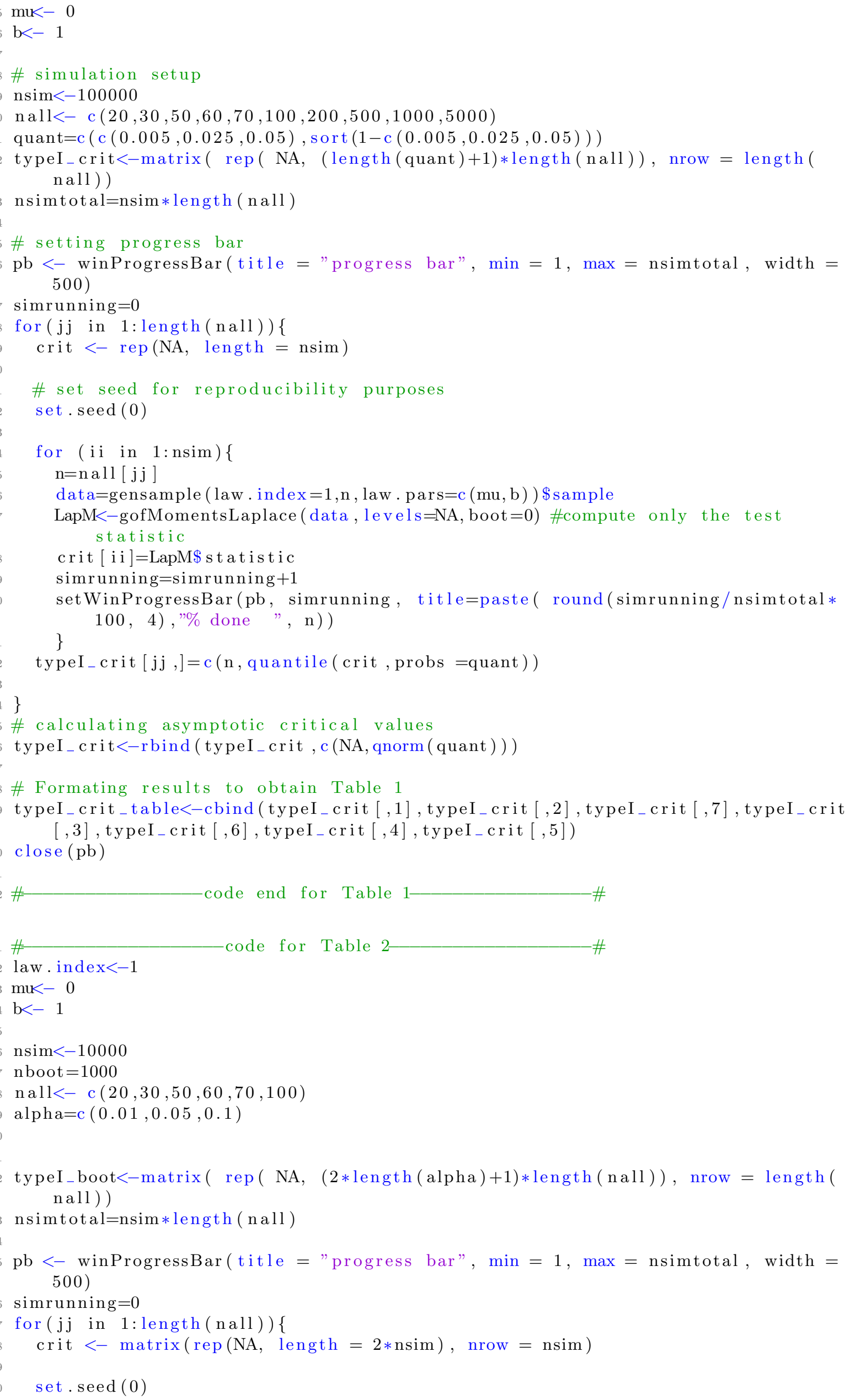




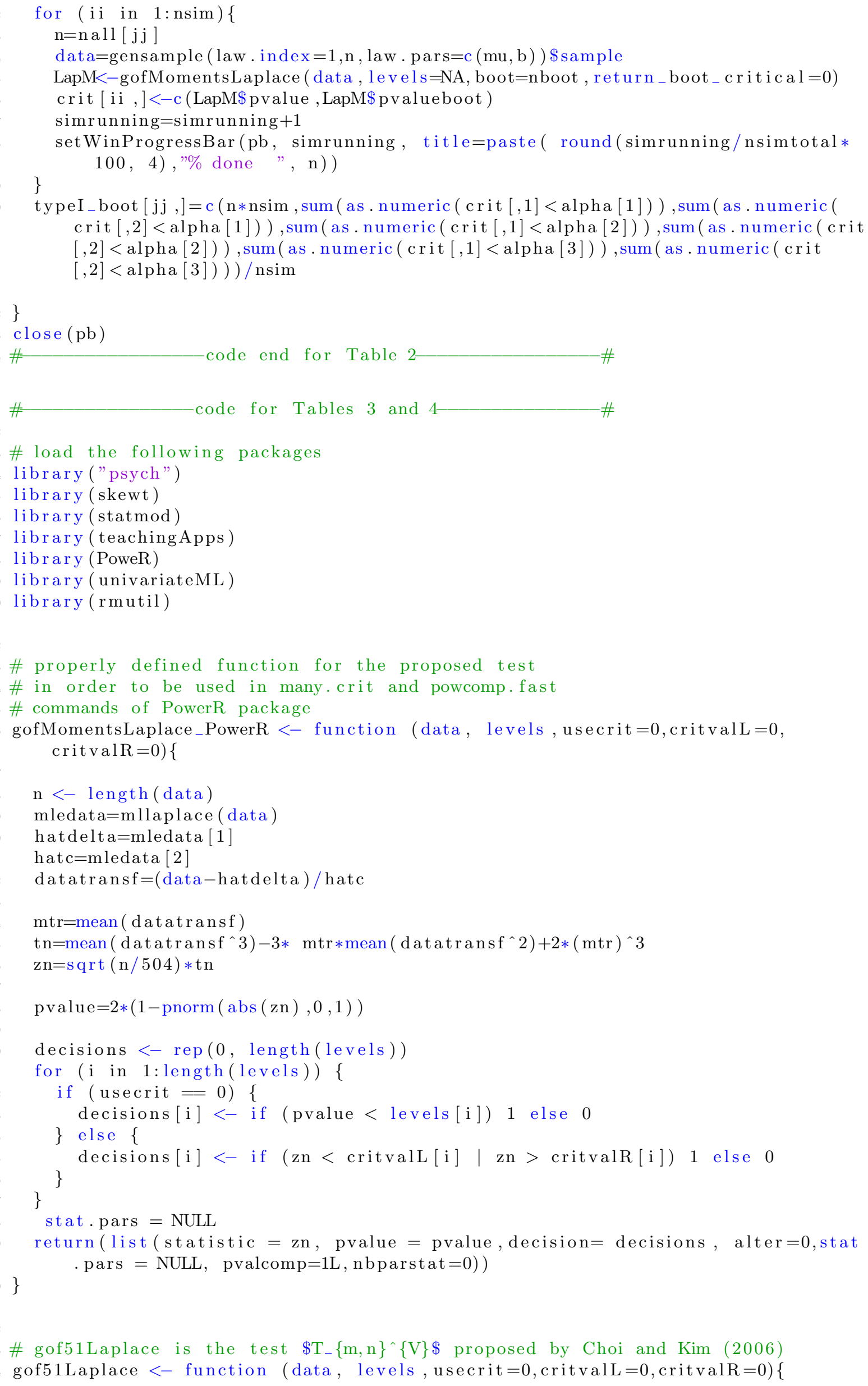




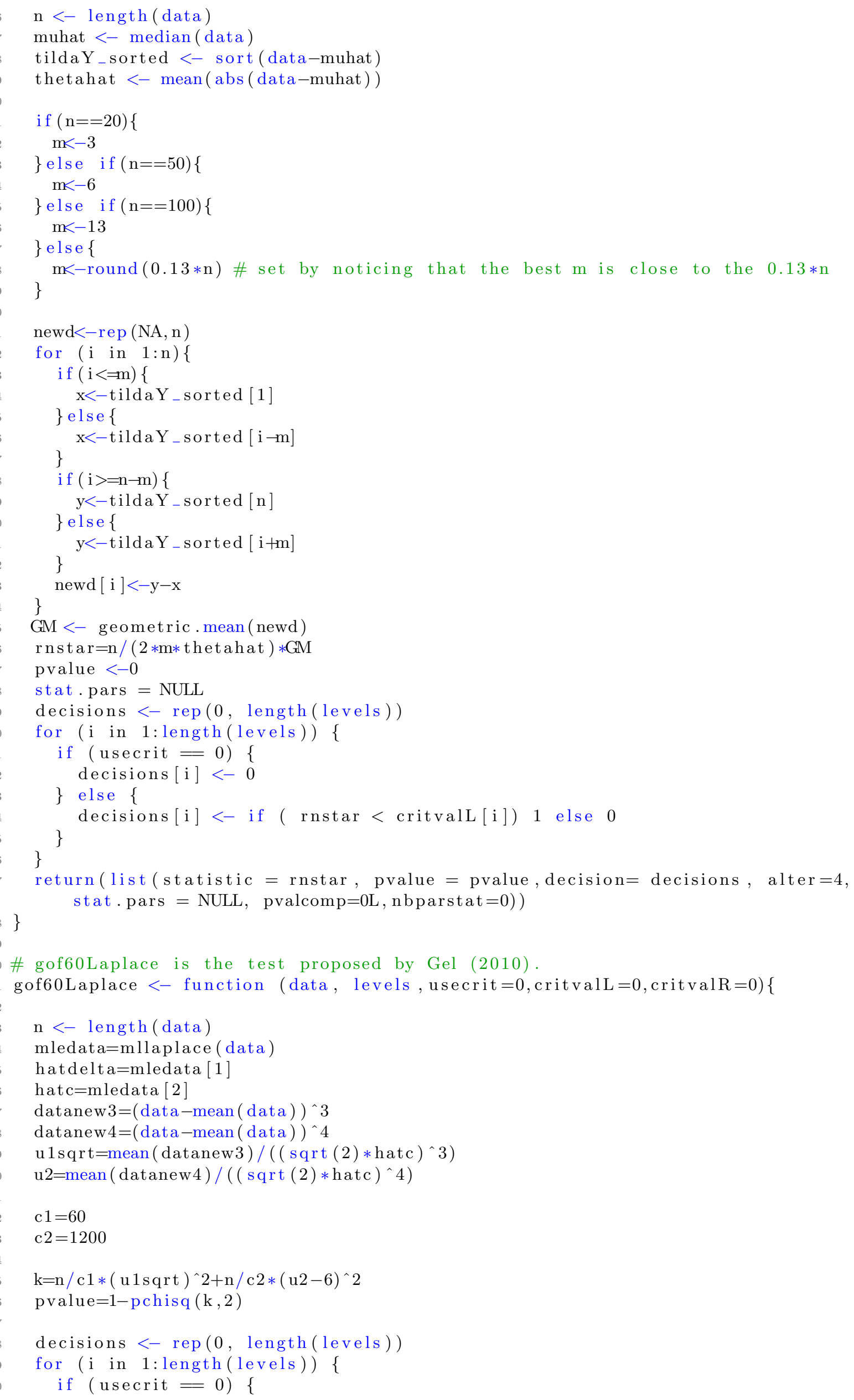




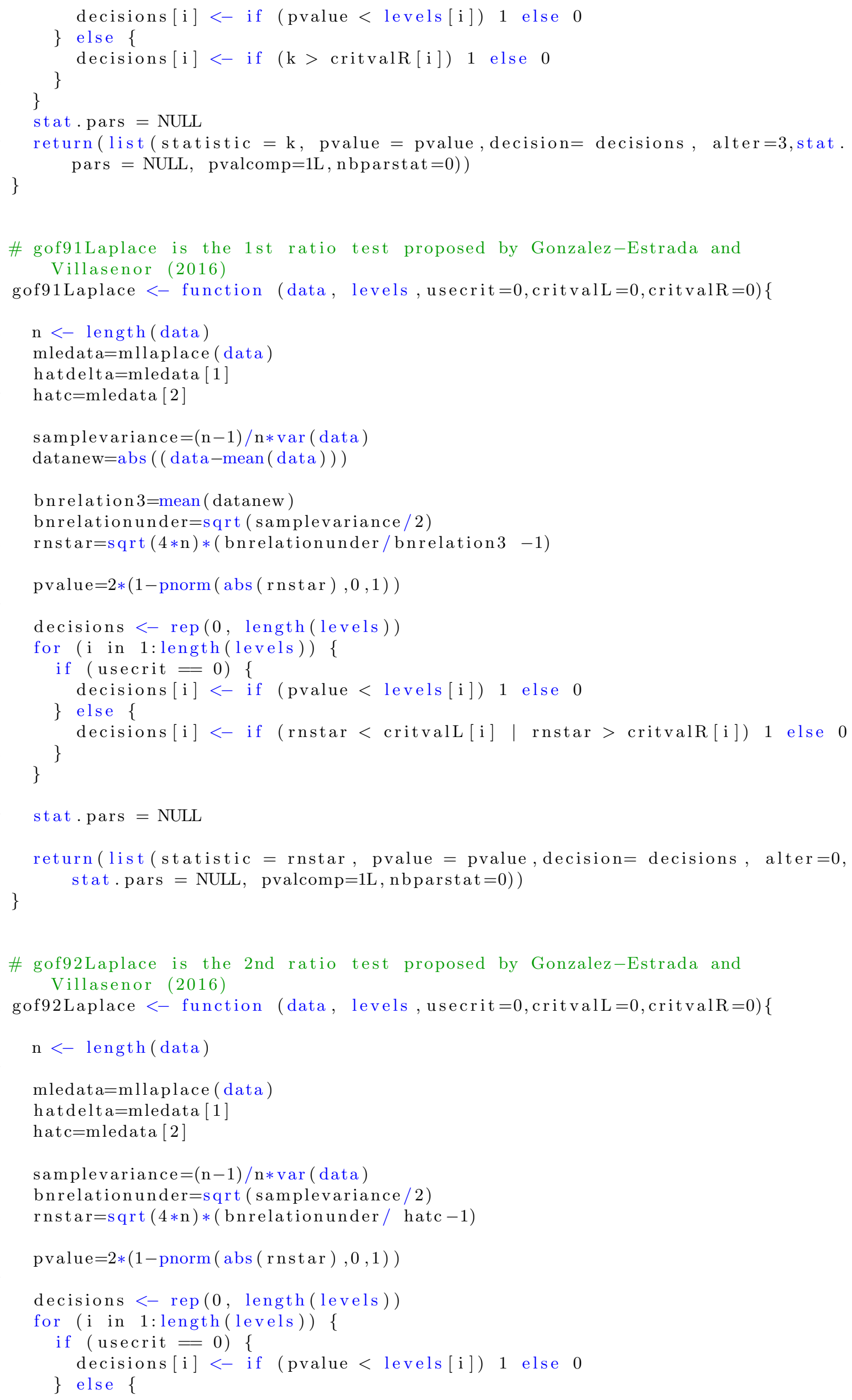




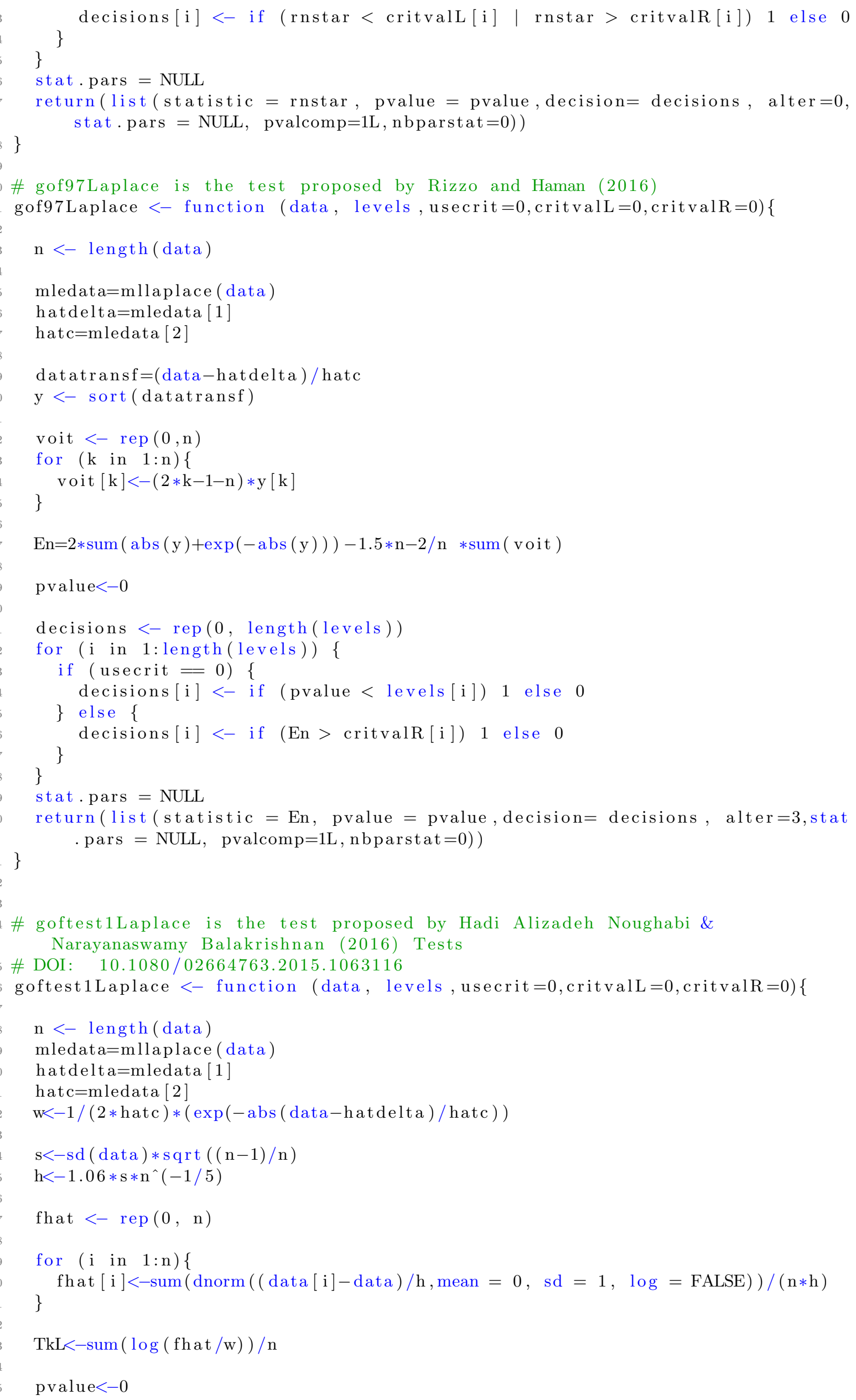




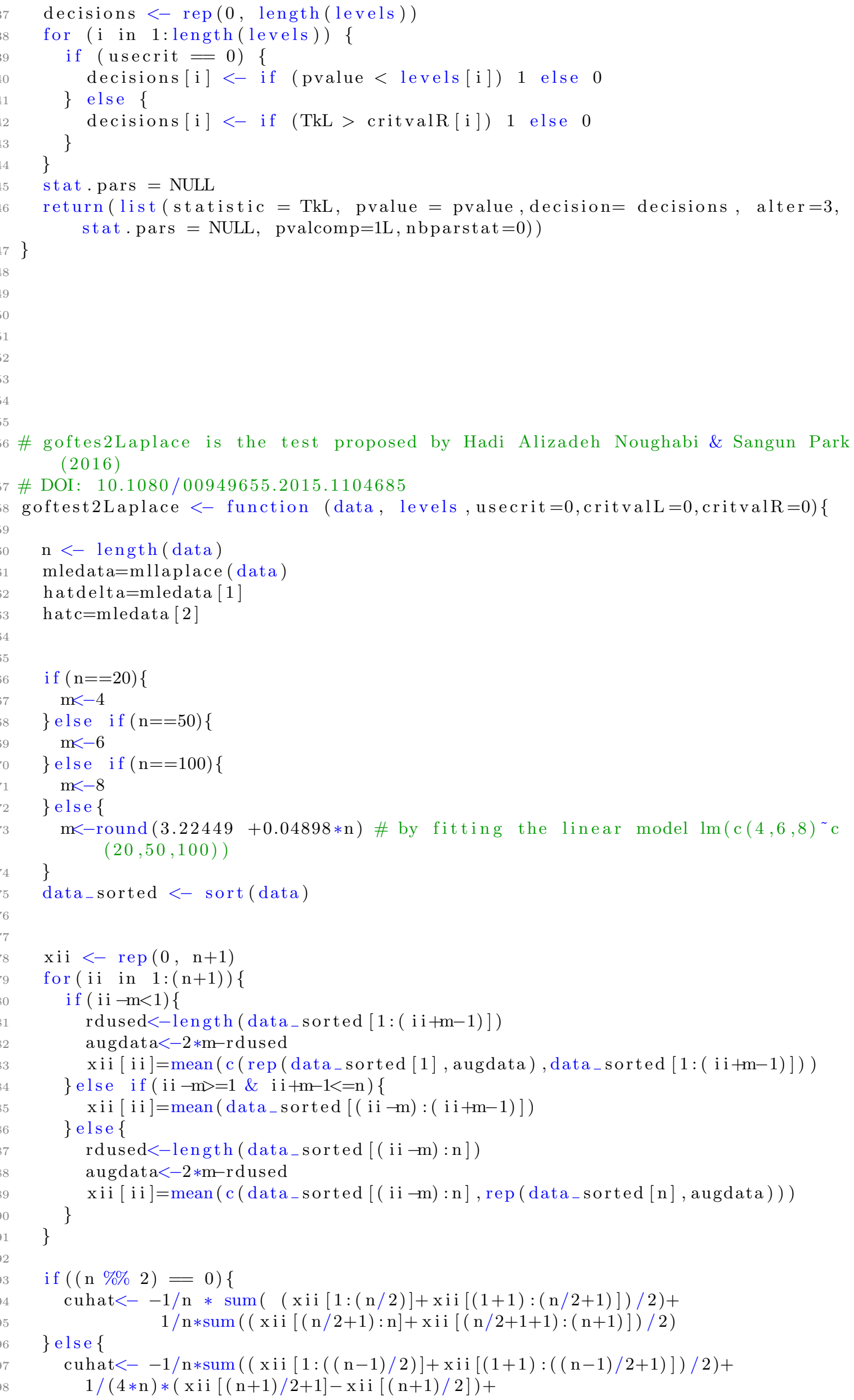




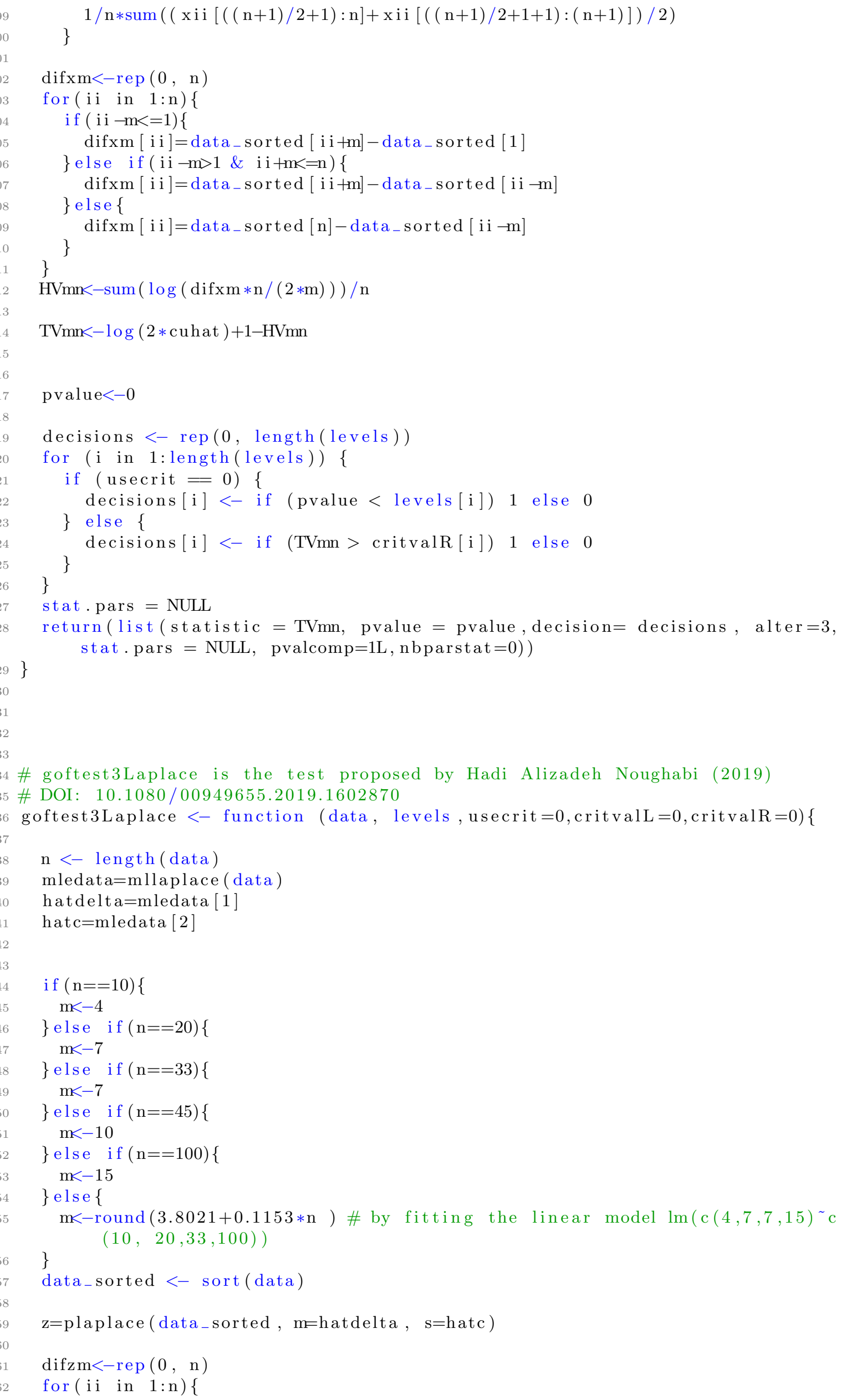




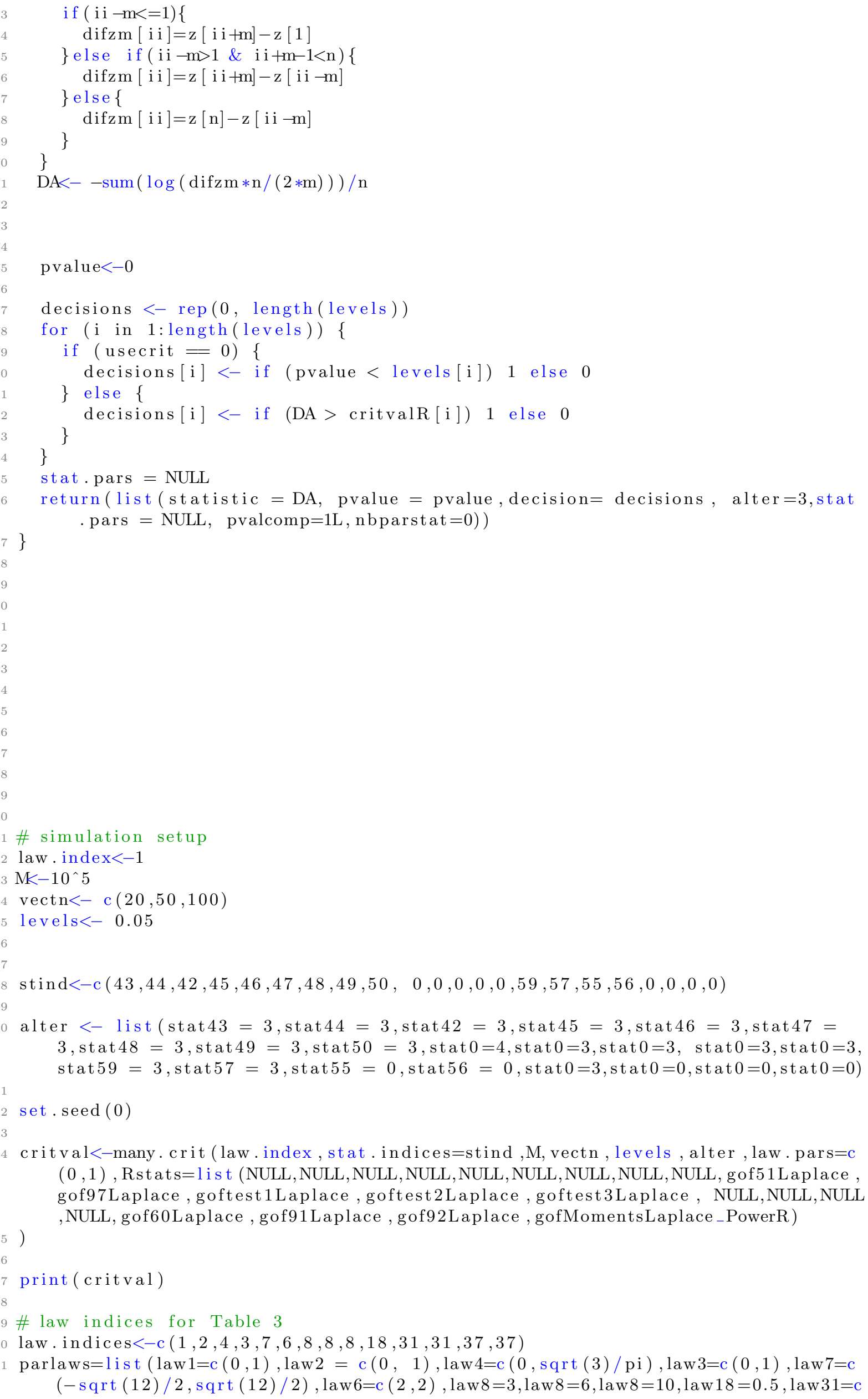




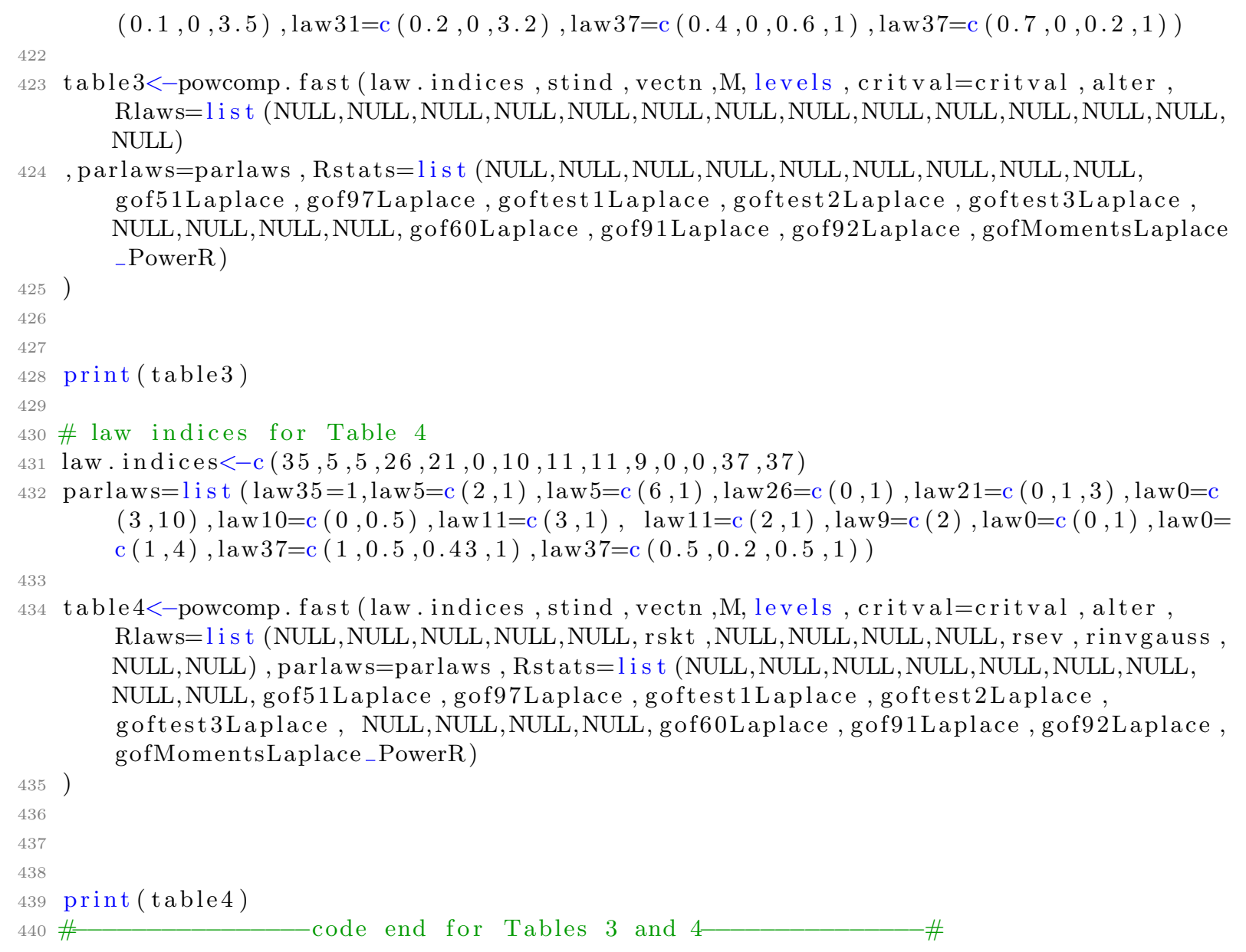

\section{Affiliation:}

Apostolos Batsidis

Department of Mathematics

University of Ioannina

Ioannina, Greece E-mail: abatsidis@uoi.gr

URL: http://users.uoi.gr/abatsidis/

Polychronis Economou

Department of Civil Engineering

University of Patras

Rion-Patras, Greece E-mail: peconom@upatras.gr

URL: http://www.des.upatras.gr/amm/economou/

Shaul K. Bar-Lev

Faculty of Industrial Engineering and Technology Management

HIT - Holon Institute of Technology

Holon, Israel

E-mail: shaulb@hit.ac.il

URL: https://www.hit.ac.il/en/faculty_staff/Shaul_Bar-Lev

\section{Austrian Journal of Statistics}

published by the Austrian Society of Statistics

Volume 51

January 2022 http://www.ajs.or.at/

http://www.osg.or.at/

Submitted: 2020-10-26

Accepted: 2021-01-15 